\title{
Actively Secure Private Function Evaluation
}

\author{
Payman Mohassel ${ }^{1,2}$, Saeed Sadeghian ${ }^{1}$, and Nigel P. Smart ${ }^{3}$ \\ 1 Dept. Computer Science, University of Calgary, \\ pmohasse@ucalgary.ca, sadeghis@ucalgary.ca \\ 2 Yahoo Labs, \\ pmohassel@yahoo-inc.com \\ 3 Dept. Computer Science, University of Bristol, \\ nigel@cs.bris.ac.uk
}

\begin{abstract}
We propose the first general framework for designing actively secure private function evaluation (PFE), not based on universal circuits. Our framework is naturally divided into pre-processing and online stages and can be instantiated using any generic actively secure multiparty computation (MPC) protocol.

Our framework helps address the main open questions about efficiency of actively secure PFE. On the theoretical side, our framework yields the first actively secure PFE with linear complexity in the circuit size. On the practical side, we obtain the first actively secure PFE for arithmetic circuits with $O(g \cdot \log g)$ complexity where $g$ is the circuit size. The best previous construction (of practical interest) is based on an arithmetic universal circuit and has complexity $O\left(g^{5}\right)$.

We also introduce the first linear Zero-Knowledge proof of correctness of "extended permutation" of ciphertexts (a generalization of ZK proof of correct shuffles) which maybe of independent interest.
\end{abstract}

Keywords. Secure Multi-Party Computation, Private Function Evaluation, Malicious Adversary, ZeroKnowledge Proof of Shuffle

\section{Introduction}

Private Function Evaluation (PFE) is a special case of Multi-Party Computation (MPC), where the parties compute a function which is a private input of one of the parties, say party $P_{1}$. The key additional security requirement is that all that should leak about the function to an adversary, who does not control $P_{1}$, is the size of the circuit (i.e. the number of gates and distinct wires within the circuit). Clearly, PFE follows immediately from MPC by designing an MPC functionality which implements a universal machine/circuit; thus the only open questions in PFE research are those of efficiency. Using universal circuits one can achieve complexity of $O\left(g^{5}\right)$ in case of arithmetic circuits 23] and $O(g \cdot \log g)$ for boolean circuits [26]. For ease of exposition we ignore the factors depending on the number of parties and the security parameters as they depend on the particular underlying MPC being used. We still provide some numbers for the specific SPDZ instantiation in section 5 .

A number of previous work [1|2|4|12|14|15|16|17|22|24 have considered the design and implementation of more efficient general- and special-purpose private function evaluation. A major motivation behind these solutions (and PFE in general) is to hide the function being computed since it is proprietary, private or contains sensitive information. Some applications of interest considered in the literature are software diagnostic [4, medical applications [2], and intrusion detection systems [20].

But all prior solutions are in the semi-honest model and fail in the presence of an active adversary who does not follow the steps of the protocol (with the exception of the generic approach of applying an actively secure MPC to universal circuits). For example, a malicious party who does not own the function can cheat to learn the proprietary function or modify the outcome of computation without the function-holders' knowledge. Or a malicious function-holder, can learn information about honest parties' inputs.

This article the full version of an earlier article: Asiacrypt 2014, (C) IACR 2014, http://dx.doi.org/10.1007/ 978-3-662-45608-8_26 
One may question the need for actively secure PFE as the function-holder can cheat and use a malicious function, which reveals information about the other party's input. While we consider the general scenario in our protocols, there are common practical scenarios where the function-holder has no output in the computation, and therefore maliciously changing the function still does not let him learn anything even if he is actively cheating.

\subsection{Our Contribution}

In this work, we present the first general framework for designing actively secure PFE, not based on universal circuits. Our framework can be instantiated upon a generic actively secure MPC protocol satisfying quite general properties; namely that they are secret sharing based, actively secure (either robust or with aborts), can implement reactive functionalities, and have an ability to open various sharings securely, as well as generate (efficiently) sharings of random values. Suitable actively secure MPC protocols include BDOZ [3] and SPDZ [8] (for the case of arithmetic circuits and an arbitrary number of players with a dishonest majority), Tiny-OT [19] (for binary circuits and two players), or protocols such as that implemented in VIFF [7] utilizing Shamir secret sharing with a threshold of $t<n / 3$.

Our framework helps address the main open questions about efficiency of actively secure PFE. On a theoretical note, we use it to show that actively secure PFE with linear complexity (in circuit size) is indeed feasible while avoiding strong primitives such as fully-homomorphic encryption (FHE) ${ }^{4}$ On a practical note, we obtain a practical actively secure PFE for arithmetic circuit with $O(g \cdot \log g)$ complexity (a significant reduction from $O\left(g^{5}\right)[23]$, and the first actively secure PFE in the information-theoretic setting.

Our Framework. Our framework can be seen as an extension of the new framework of [17] which is only secure against passive adversaries. The key idea in [17] is to divide the problem into two sub-problems, the problem of hiding the topology of the wiring between individual gates (topology hiding), and the problem of hiding exactly what gate is evaluated (gate hiding), i.e. an addition or a multiplication (or AND/OR/XOR in case of boolean circuits).

This framework yields better asymptotic and practical efficiency for passively secure PFE compared to the universal circuit approach (see [17] for a detailed efficiency comparison). An important open question is then how to extend their solution to the case of active adversaries efficiently. In this paper we do exactly that by providing a recipe for turning any actively secure MPC protocol that satisfies our general requirements into an actively secure PFE protocol.

Our framework operates in two phases, an offline phase and an online phase. As in the case of standard MPC in the pre-processing model, our offline phase is input independent but it depends on the function. The offline phase is use-once, in the sense that the data produced cannot be reused for multiple invocations of the online phase. We note that a similar function-dependent pre-processing model (referred to as dedicated pre-processing) was recently considered in 9. Dedicated pre-processing is particularly natural in PFE applications where the sensitive/proprietary function stays fixed for a period of time and is used in multiple executions (clearly in the latter case we need to execute the pre-processing multiple times, but this can be done in advance). Of course, if one is not willing to count a function-dependent offline phase as valid, then our complexities would be the combination of the two phases. It maybe the case that our underlying MPC protocol is itself in the pre-processing model (e.g. 38|19), in which case that pre-processing will be essentially independent of the input and function being evaluated. Our framework shows the feasibility of offline computation independent of inputs, which was not the case in [17. We elaborate on the two phases next:

Offline Phase. Roughly speaking, our offline phase generates two vectors of random values, maps the second to a new vector using a mapping that captures the topology of the circuit (referred to as extended permutation

\footnotetext{
${ }^{4}$ Note that with the use of the right circuit-private FHE scheme [21, and appropriate ZK proofs for correctness of the computation on encrypted data, it is likely possible to achieve linear PFE based on FHE, but we are interested in the use of much weaker primitives such as singly homomorphic encryption.
} 
in [17), and subtracts the result from the first. The result of the subtraction (difference vector) is opened while the two original vectors are shared among the parties. The two random vectors are used as one-time pads of all the intermediate values in the circuit, while the "difference vector" is used by the function-holder to connect the output of one gate to the input of another without learning the values or revealing the circuit topology. The offline phase also generates one-time MACs of all the components of the "difference vector" computed above, using a fixed global MAC key. These MACs are used to check the function-holder's work in the online phase of the protocol. These steps commit $P_{1}$ privately to the topology of the circuit. We also privately commit $P_{1}$ to gate types, hence fully committing him to the function being computed.

Online Phase. Our online, or circuit evaluation, phase is very distinct from that deployed in the underlying MPC protocol we use. In existing instantiations of our underlying MPC protocol, parties evaluate gates on values whose secrecy is maintained due to the fact that one is working on secret shared values only. In our protocol the parties have public one-time pad encryptions of the values being computed on, but the encryption keys, which are the random values generated in the offline phase, remain secret-shared. Party $P_{1}$ (the function holder) then uses the random vectors computed in the offline phase to transform the encrypted output of one gate to the encrypted input of the upcoming gate while maintaining one-time MACs of all the values he computes. These MACs allow all other parties to check $P_{1}$ 's work without learning the circuit topology. These operations are carried out securely using the underlying MPC protocol.

In both the online and the offline phase, all parties check $P_{1}$ 's work by checking the MACs of the values he computes locally. If any of the MACs fail, in case of security with abort, parties can simply end the protocol. But in case of robust MPC (e.g. $t<n / 3$ for robust information theoretically secure protocols) the protocol needs to continue without $P_{1}$. To achieve this, honest parties jointly recover $P_{1}$ 's function and play his role in the remainder of the protocol.

In our protocols, if any adversary deviates from the protocol then, except with negligible probability, the honest parties will either abort, or be able to recover from the introduced error. The exact response depends on the underlying MPC protocol on which our PFE protocol is built. In all cases the privacy of the honest players inputs is preserved, bar what can be obtained from the output of the private function chosen by player $P_{1}$. Note that $P_{1}$ may or may not be a recipient of output, but many application of PFE are concerned with scenarios where the function-holder has no output.

Efficient Instantiations. One can efficiently instantiate our online phase with a linear complexity, using any actively secure MPC satisfying our requirements. The main challenge, therefore, lies in efficient instantiation of the offline phase. It is possible to implement our offline phase using any actively secure MPC sub-protocol as well (by securely computing a circuit that performs the above mentioned task) but the resulting constructions would neither be linear nor constant-round.

- We introduce a instantiation with $O(g)$ complexity, proving the feasibility of linear actively secure PFE for the first time. Our main new technical ingredient is a linear zero-knowledge (ZK) proof of "correct extended permutation" of ElGamal ciphertexts. While linear ZK proofs of shuffles are well-studied, it is not clear how to extend the techniques to extended permutation (see our incomplete attempt in Appendix B Instead, we propose a generic and linear solution that uses ZK proof of a correct shuffle in a black-box manner, and may be of independent interest. Our solution is based on the switching network construction of EP [17. This construction consists of three components, two of which are permutation networks. Instead of evaluating switches, we use singly homomorphic encryption to evaluate each component, and then re-randomize. We use existing ZK proofs of shuffle to prove the correctness of first and third components which perform permutation. The middle component requires a separate compilation of ZK protocols. Note that generically applying ZK proofs to UC circuit evaluation does not provide a linear solution, and applying ZK proofs for the EP component also does not work. Our customized linear $\mathcal{Z} \mathcal{K}_{\mathrm{EP}}$ gets around these problems.

- We introduce a constant-round instantiation with $O(g \cdot \log g)$ complexity (contrast with $O\left(g^{5}\right)$ complexity for universal arithmetic circuits) that is also of practical interest. Our technique is itself an extension of ideas from [17. In particular the basic algorithm is that of [17] for oblivious evaluation of a switching 
network, but some care needs to be taken to make sure the protocol is actively secure. This is done by applying MACs to the data being computed on. However, instead of having the MAC values being secret shared (as in SPDZ) or kept secret (as in BDOZ and Tiny-OT), the MAC values are public with the keys remaining secret shared. Nevertheless, the MACs used are very similar to those used in the BDOZ and Tiny-OT protocols [319, since they are two-key MACs in which one key is a per message key and one is a global key. While using MAC's is quite standard for ensuring consistency of data, our efficient deployment in the framework is non-trivial and novel. For example, while addition of MACs in the offline phase is done using a generic MPC, the circuit evaluation (online phase) does not use an MPC. This is different from [17]'s approach and previous MPC work. General active security techniques can not be directly employed in this context. It is not clear how to use cut-and-choose in case of PFE, e.g. it is not clear how not to reveal the function in the opening, and there are additional components (i.e. EP) in a PFE protocol which cut-and-choose does not seem to resolve.

Efficiency Discussion. We emphasize that our linear complexity solution is a feasibility result at it was an open question whether active PFE with linear complexity in circuit size is possible given simple crypto primitive such as singly homomorphic encryption (as opposed FHE). Our "efficient" arithmetic PFE only requires $O(g \log g)$ multiplication gates and it is a significant improvement in comparison with applying of arithmetic MPC to universal arithmetic circuit of size $O\left(g^{5}\right)$ [23. If we apply active secure MPC for arithmetic circuits to this universal circuit the complexity cannot get better than $O\left(g^{5}\right)$. One can turn an arithmetic circuit into a boolean circuit and use Valiant's boolean UC [26] to obtain a PFE. But this is highly inefficient, and therefore we do not discuss this in detail.

\section{Notation and the Underlying MPC Protocol}

We assume our function $f$ to be evaluated will eventually be given by player $P_{1}$ as an arithmetic circuit over a finite field $\mathbf{F}_{p}$; note $p$ may not necessarily be prime. We let $\mathbf{g}(f)$ denote the number of gates in the circuit representing $f$. For gates with fan-out greater than one, we count each seperate output wire as a different wire. We also select a value $k$ such that $p^{k}>2^{\text {sec }}$, where sec is the security parameter; this is to ensure security of our MAC checking procedure in the online phase.

We assume $n$ parties $P_{1}, \ldots, P_{n}$, of which an adversary may corrupt (statically) up to $t$ of them; the value of $t$ being dependent on the specific underlying MPC protocol. The corrupted adversaries could include party $P_{1}$. The MPC protocol should implement the functionality described in Figure 1 . This functionality is slightly different from standard MPC functionalities in that we try to capture both the honest majority and the dishonest majority setting; and in the latter setting the adversary can force the functionality to abort at any stage of the computation and not just the output. We also introduce another operation called Cheat which will be useful in what follows.

It is clear that modern actively secure MPC protocols such as [7/8/19, implement this functionality in different settings. Thus various different settings (i.e. different values of $n, p$ and $t$ ) will be able to be dealt with in our resulting PFE protocol by simply plugging in a different underlying MPC protocol. To ease exposition later we express our MPC protocol as evaluating functions in the finite field $\mathbf{F}_{p^{k}}$. Clearly such an MPC protocol can be built out of one which evaluates functions over the base finite field $\mathbf{F}_{p}$.

To ease notation in what follows we shall let $[$ varid $]$ denote the value stored by the functionality under (varid, $a$ ); and will write $[z]=[x]+[y]$ as a shorthand for calling Add and $[z]=[x] \cdot[y]$ as a shorthand for calling Multiply. And by abuse of notation we will let varid denote the value, $x$, of the data item held in location (varid, $x)$.

\section{Our Active PFE Framework}

In this section we describe our active PFE framework in detail. We start by describing the offline functionality which pre-processes the function/circuit the parties want to compute (Section 3.1). Then, in Section 3.2 . 
Functionality $\mathcal{F}_{\mathrm{MPC}}$

The functionality consists of seven externally exposed commands Initialize, Cheat, Input Data, Random, Add, Multiply, and Output and one internal subroutine Wait.

Initialize: On input (init, $p, k$, flag) from all parties, the functionality activates and stores $p$ and $k$; and a representation of $\mathbf{F}_{p^{k}}$. The value of flag is assigned to the variable dhm, to signal whether the MPC functionality should operate in the dishonest majority setting. The set of "valid" players is initially set to all players. In what follows we denote the set of adversarial players by $\mathcal{A}$.

Cheat: This is a command which takes as input a player index $i$, it models the case of (most) robust MPC protocols in the honest majority case. On execution the functionality aborts if $\mathrm{dhm}$ is set to true. Otherwise the functionality waits for input from all players. If a majority of the players return $O K$ then the functionality reveals all inputs made by player $i$, and player $i$ is removed from the list of "valid" players (the functionality continues as if player $i$ does not exist).

Wait: This does two things depending on the value of $\mathrm{dhm}$.

- If $\mathrm{dhm}$ is set to true then it waits on the environment to return a $G O / N O-G O$ decision. If the environment returns $\mathrm{NO}-\mathrm{GO}$ then the functionality aborts.

- If $\mathrm{dhm}$ is set to false then it waits on the environment. The environment will either return $G O$, in which case it does nothing, or the environment returns a value $i \in \mathcal{A}$, in which case Cheat $(i)$ is called.

Input Data: On input (input, $P_{i}$, varid,$\left.x\right)$ from $P_{i}$ and (input, $P_{i}$, varid, $?$ ) from all other parties, with varid a fresh identifier, the functionality stores (varid, $x$ ). The functionality then calls Wait.

Random: On command (random, varid) from all parties, with varid a fresh identifier, the functionality selects a random value $r$ in $\mathbf{F}_{p^{k}}$ and stores (varid, $r$ ). The functionality then calls Wait.

Add: On command (add, varid 1, varid $_{2}$, varid va $_{3}$ ) from all parties (if varid $_{1}$, varid $_{2}$ are present in memory and varid $_{3}$ is not), the functionality retrieves $\left(\right.$ varid $\left._{1}, x\right),\left(\right.$ varid $\left._{2}, y\right)$ and stores $\left(\right.$ varid $\left._{3}, x+y\right)$. The functionality then calls Wait.

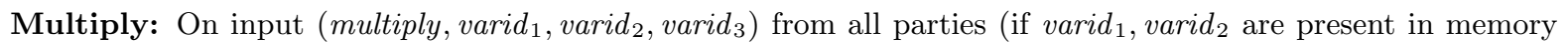
and varid $_{3}$ is not), the functionality retrieves $\left(\operatorname{varid}_{1}, x\right),\left(\operatorname{varid}_{2}, y\right)$ and stores $\left(\operatorname{varid}_{3}, x \cdot y\right)$. The functionality then calls Wait.

Output: On input (output, varid) from all honest parties (if varid is present in memory), the functionality retrieves (varid, $x$ ) and outputs it to the environment. The functionality then calls Wait, and only if Wait does not abort then it outputs $x$ to all players.

Fig. 1: The required ideal functionality for MPC 
we show that given a secure implementation of $\mathcal{F}_{\text {OfFuINE }}$, one can efficiently (linear complexity) construct an actively secure PFE based on any actively secure MPC. We postpone efficient instantiations of $\mathcal{F}_{\text {Offline }}$ to later sections.

\subsection{The Function Pre-Processing (Offline) Phase}

In this section we detail the requirements of our pre-processing step once player $P_{1}$ has decided on the function $f$ to be evaluated. $P_{1}$ is only required to enter a valid circuit, equivalent to his function $f$ into the protocol. Each non-output wire $w$ in the circuit is connected at one end (which we shall call the outgoing wire or left point) to a source, this is either the output of a (non-output) gate or an input wire. Conversely each non-output wire is connected at the other end (which we shall call the incoming wire or right point) to a destination point which is always an input to a gate. We denote the number of distinct Incoming Wires on the right by $\operatorname{iw}(f)$. We let $\mathrm{w}(f)$ denote the number of Outgoing Wires on the left. Note that $\operatorname{iw}(f)=2 g$ and $\mathrm{ow}(f)=n+g-o$ where $o$ is the number of output gates in the circuit. Since we are dealing with arbitrary fan out we have that $\operatorname{ow}(f) \leq \operatorname{iw}(f)$.

Functionality $\mathcal{F}_{\text {OffLine }}$

Initialize: As for $\mathcal{F}_{\mathrm{MPC}}$.

Wait: As for $\mathcal{F}_{\mathrm{MPC}}$.

Input Data: As for $\mathcal{F}_{\mathrm{MPC}}$.

Cheat: As for $\mathcal{F}_{\mathrm{MPC}}$.

Random: As for $\mathcal{F}_{\mathrm{MPC}}$.

Add: As for $\mathcal{F}_{\mathrm{MPC}}$.

Multiply: As for $\mathcal{F}_{\mathrm{MPC}}$.

Output: As for $\mathcal{F}_{\mathrm{MPC}}$.

Input Function: On input (inputfunction, $\pi, f$ ) from player $P_{1}$ the functionality performs the following operations

- The functionality calls (random, $K$ ).

- If $f$ is not a valid arithmetic circuit then the functionality aborts.

- For $i \in\{1, \ldots, \operatorname{iw}(f)\}$ the functionality calls $\left(\right.$ random, $\left.r_{i}\right)$ and $\left(\right.$ random, $\left.s_{i}\right)$.

- For $j \in\{1, \ldots$, ow $(f)\}$ the functionality calls $\left(\right.$ random,$\left.l_{j}\right)$ and $\left(\right.$ random, $\left.t_{j}\right)$.

- The functionality then computes, for all $i \in\{1, \ldots, \operatorname{iw}(f)\}$

$$
\left[p_{i}\right]=\left[r_{i}\right]-\left[\ell_{\pi(i)}\right], \quad\left[q_{i}\right]=\left(\left[s_{i}\right]-\left[t_{\pi(i)}\right]\right)+\left(\left[r_{i}\right]-\left[\ell_{\pi(i)}\right]\right) \cdot[K]
$$

- The functionality then outputs $\left(p_{i}, q_{i}\right)$ to all players, for $i \in\{1, \ldots, \operatorname{iw}(f)\}$, by calling (output, $\left.p_{i}\right)$ and (output, $q_{i}$ ).

- For $i \in\{1, \ldots, g\}$ the functionality calls (input, $\left.P_{1}, G_{i}, 0\right)$ if gate $i$ in the description of $f$ is an addition gate, and (input, $\left.P_{1}, G_{i}, 1\right)$ if gate $i$ is a multiplication gate.

Fig. 2: The required ideal functionality for the Offline Phase

To fully capture the topology of the circuit we give each outgoing wire and incoming wire in the circuit a unique label. The labels for the outgoing wires will be $\{1, \ldots$, ow $(f)\}$ starting from the input wires and then moving to the output wires of each gate in a topological order decided by $P_{1}$, whilst the labels for the incoming wires will be $\{1, \ldots, \operatorname{iw}(f)\}$ labelling the input wires to each gate in the same topological order. The topology is then defined by a mapping from outgoing wires to incoming wires and is called an "extended permutation" in [17 as demonstrated in Figure 3. We denote the inverse of this mapping by a function $\pi$ from $\{1, \ldots, \operatorname{iw}(f)\}$ onto $\{1, \ldots$, ow $(f)\}$. If $w$ is a wire in the circuit with incoming wire label $i$, then it's outgoing wire label is given by $j=\pi(i)$.

To execute the function pre-processing, player $P_{1}$ on input of $f$ determines a mapping $\pi$ corresponding to $f$. The offline phase functionality $\mathcal{F}_{\text {OfFLINE }}$ which is described in Figure 2 extends the $\mathcal{F}_{\text {MPC }}$ functionality 


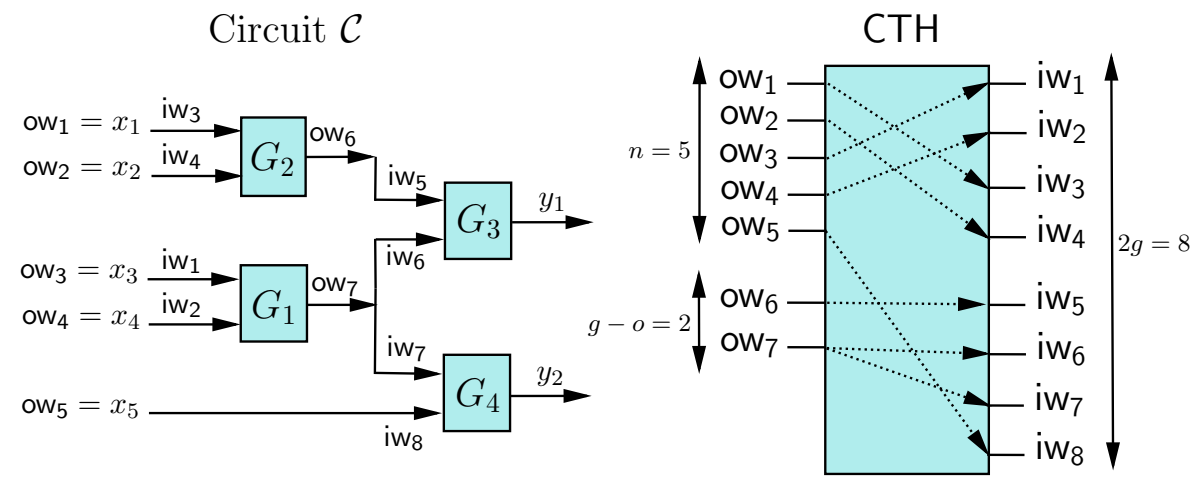

Fig. 3: An example circuit and the corresponding mapping [17

of Figure 1 by adding an additional operation Input Function. The Input Function generates a vector of random (but correlated) values and their one-time MACs using a fixed global MAC key $K$. In particular, the functionality first stores a vector of random values $\left(r_{i}\right)$ for each incoming wire and another vector of random values $\left(\ell_{i}\right)$ for the outgoing wires in the circuit. These random values will play the role of "pads" for one-time encryption of the computed wire values in the online phase. The functionality then computes $p_{i}$, the difference between each outgoing wire's value $r_{i}$ and the corresponding incoming wires' value $\ell_{\pi(i)}$, and reveals $p_{i}$ to all parties. This difference vector will allow $P_{1}$ to maintain one-time encryption of each wire value in the online phase without revealing the circuit topology. Additional random values $\left(s_{i}, t_{i}\right)$ and the global MAC key $K$ are used to compute one-time MACs of each $p_{i}$, namely $q_{i}$. These MACs will be used to check $P_{1}$ 's actions in the online phase. The Input Function also commits $P_{1}$ to the function of each gate in his circuit by storing a bit ( 0 for addition and 1 for multiplication) for each gate.

\subsection{The Function Evaluation (Online) Phase}

We can now present our framework for actively secure PFE. We wish to implement the functionality in Figure 4. We express the functionality as evaluating a function $f$ provided by $P_{1}$ which takes as input $n$ inputs in $\mathbf{F}_{p^{k}}$, one from each player. Again we present the functionality in both the honest majority and the dishonest majority settings.

Realizing $\mathcal{F}_{\text {Online }}$ Given $\mathcal{F}_{\text {Offline }}$ and $\mathcal{F}_{\text {MPC }}$ A generic instantiation of $\mathcal{F}_{\text {OfFLINE }}$ based on any MPC is give in Figure 6. The idea is to work with one-time pad encryptions of the values for all intermediate wires and the corresponding one-time MACs. Here, the pads ( $r, \ell, s, t$ values), as well as the MAC Key $K$ are generated by the offline functionality, and shared among the parties so no party can learn intermediate values or forge MACs on his own.

In more detail, the protocol proceeds as follows. Initially, parties compute one-time encryption of the input values to the circuit (pads are the corresponding $\ell$ values). Then, the following process is repeated for every gate in the circuit until every gate is processed. Parties then open the outcome of the output gates as their final result.

For each gate, party $P_{1}$ uses the "difference vectors" ( $p_{i}$ values) from the offline phase to transform the one-time encryption of output of the previous gate to the one-time encryption of input of the current gate (the result is denoted by $d_{i_{0}}, d_{i_{1}}$ for the $i$-th gate.), without revealing the topology or learning the actual wire values. This is diagrammatically presented in Figure 5 to aid the reader. A similar transformation is done on MACs of the wire values (using $q_{i}$ values) in order to keep $P_{1}$ honest in his computation (denoted by $\left.m_{i_{0}}, m_{i_{1}}\right)$.

Then, the protocol proceeds by jointly removing the one-time pads for the two inputs of the current gate and evaluating it together in order to compute a shared output $z_{i}$. Note that in this gate evaluation the gate 
Initialize: On input (init, $p, k, f l a g$ ) from all players, the functionality activates and stores $p$ and $k$; and a representation of $\mathbf{F}_{p^{k}}$. The value of flag is assigned to the variable $\mathrm{dhm}$, to signal whether the underlying MPC functionality should operate in the dishonest majority setting.

Wait: If $\mathrm{dhm}$ is set to false then this does nothing. Otherwise it waits on the environment to return a GO/NO-GO decision. If the environment returns $N O-G O$ then the functionality aborts.

Input Function: On input (inputfunction, $f$ ) from player $P_{1}$ the functionality stores (function, $f$ ). The functionality now calls Wait.

Input Data: On input (input, $\left.P_{i}, x_{i}\right)$ from player $P_{i}$ the functionality stores (input, $i, x_{i}$ ). The functionality now calls Wait.

Output: On input (output) from all honest players the functionality retrieves the data $x_{i}$ stored in $\left(\right.$ input, $\left.i, x_{i}\right)$ for $i \in\{1, \ldots, n\}$ (if all do not exist then the functionality aborts). The functionality then retrieves $f$ from (function, $f$ ) and computes $y=f\left(x_{1}, \ldots, x_{n}\right)$ and outputs it to the environment (or aborts if (function, $f$ ) has not been stored). The functionality now calls Wait. Only on a successful return from Wait will the functionality output $y$ to all players.

Fig. 4: The required ideal functionality for PFE

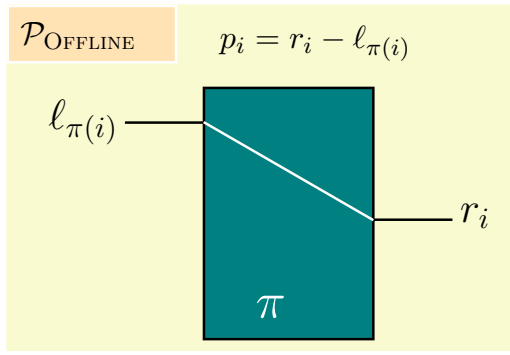

$$
\begin{aligned}
& \mathcal{P}_{\text {OnLINe }} \\
& \begin{array}{l}
\text { 1. Prepare outgoing wire } \\
u_{\pi(i)}=x_{\pi(i)}+\ell_{\pi(i)} \\
\text { 2. } P_{1} \text { computes the incomming wires' } \\
\quad d_{i}=u_{\pi(i)}+p_{i} \\
d_{i}=x_{\pi(i)}+\ell_{\pi(i)}+r_{i}-\ell_{\pi(i)} \\
d_{i}=x_{\pi(i)}+r_{i}
\end{array}
\end{aligned}
$$

Fig. 5: Transformation of one-time encryption of an outgoing wire to the one-time encryption of an incoming wire using the values computes in $\mathcal{P}_{\text {OfFLINE }}$ protocol.

type $G_{i}$ is secret and shared among the players. This step can be performed using the $\mathcal{F}_{\text {MPC }}$ operations. Then, parties compute a one-time encryption of $z_{i}$ using the corresponding $\ell$ value as the pad, and denote the result by $u_{j}$, just a relabeling where $j$ is the outgoing wire's label of the output wire of the gate (note that $j=n+i$ since the outgoing wires are labeled starting with the $n$ input wires and then the output wire of each gate).

Note, that if $P_{1}$ tries to deviate from the protocol in his local computation (i.e. when he connects outgoing wires to incoming wires) the generated MACs will not pass the jointly performed verifications and he will be caught. In that case, either the protocol aborts (in the case of dishonest majority) or his input (i.e. the function) is revealed (in the case of honest majority).

This leads to the following theorem, whose proof is given in Appendix $\mathrm{F}$

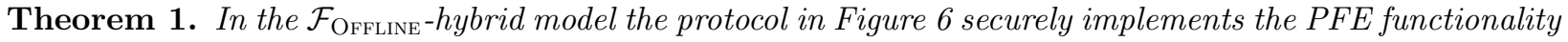
in Figure 4, with complexity $O(g)$.

\section{Implementing $\mathcal{F}_{\text {Offline }}$ with Linear Complexity}

In this section we give a linear instantiation of the offline phase of the framework. Since our online phase has linear complexity, a linear offline phase implementation leads to a linear actively secure PFE. The main challenge in obtaining a linear solution is to design a linear method for applying the extended permutation 


\section{Protocol $\mathcal{P}_{\text {OnLINE }}$}

The protocol is described in the $\mathcal{F}_{\text {OFFLINE-hybrid model. }}$

Input Function: Player $P_{1}$ given $f$ selects the switching network mapping $\pi$ and then calls (inputfunction, $\pi, f$ ) on the functionality $\mathcal{F}_{\text {Offline }}$.

Input Data: On input $\left(\right.$ input $\left., P_{i}, x_{i}\right)$ from player $P_{i}$ the protocol executes the (input, $i, x_{i}$ ) operation of the functionality $\mathcal{F}_{\text {Offuine }}$.

Output: The evaluation of the function proceeds as follows; where for ease of exposition we set $x_{\pi(h)}=y_{h}$ for all $h$, i.e. if a wire has input $x_{i}$ on the left (as outgoing wire) then it has the same value $y_{h}$ on the right (as incoming wire) where $i=\pi(h)$

\section{- Preparing Inputs to the Circuit:}

- For each input wire $i(1 \leq i \leq n)$ the players execute $\left[u_{i}\right]=\left[x_{i}\right]+\left[\ell_{i}\right]$, where $i$ is the outgoing wire's label corresponding to that input wire, and $\left[v_{i}\right]=\left[t_{i}\right]+\left(\left[x_{i}\right]+\left[\ell_{i}\right]\right) \cdot[K]$ using the $\mathcal{F}_{\text {MPC functionality }}$ available via $\mathcal{F}_{\text {OfFLINE. }}$

- Parties then call (output, $\left.u_{i}\right)$ and $\left(\right.$ output, $\left.v_{i}\right)$ to open $\left[u_{i}\right]$ and $\left[v_{i}\right]$.

- Evaluating the Circuit: For every gate $1 \leq i \leq g$ in the circuit players execute the following (here we assume that the gates are indexed in the same topological order $P_{1}$ chose to determine $\pi$ ):

- $P_{1}$ Prepares the Two Inputs for Gate $i$.

* Note that the two input wires for gate $i$ have incoming wire labels $i_{0}=2 i-1$ and $i_{1}=2 i$, and the $(u, v)$ value for their corresponding outgoing wire labels are already determined, i.e. $u_{\pi\left(i_{j}\right)}$ and $v_{\pi\left(i_{j}\right)}$ are already opened for $j \in\{0,1\}$.

* Player $P_{1}$ computes, for $j=0,1$,

$$
\begin{aligned}
d_{i_{j}}=u_{\pi\left(i_{j}\right)}+p_{i_{j}} \doteq & \left(y_{i_{j}}+\ell_{\pi\left(i_{j}\right)}\right)+\left(r_{i_{j}}-\ell_{\pi\left(i_{j}\right)}\right) \\
\doteq & y_{i_{j}}+r_{i_{j}}, \\
m_{i_{j}}=v_{\pi\left(i_{j}\right)}+q_{i_{j}} \doteq & \left(t_{\pi\left(i_{j}\right)}+\left(y_{i_{j}}+\ell_{\pi\left(i_{j}\right)}\right) \cdot K\right) \\
& +\left(\left(s_{i_{j}}-t_{\pi\left(i_{j}\right)}\right)+\left(r_{i_{j}}-\ell_{\left.\pi\left(i_{j}\right)\right)}\right) \cdot K\right) \\
\doteq & s_{i_{j}}+\left(y_{i_{j}}+r_{i_{j}}\right) \cdot K .
\end{aligned}
$$

* Player $P_{1}$ then broadcasts the values $d_{i_{j}}$ and $m_{i_{j}}$ to all players.

- Players Check $P_{1}$ 's Input Preparation.

* All players then use the $\mathcal{F}_{\text {MPC }}$ operations available (via the interface to the $\mathcal{F}_{\text {OffLINE }}$ functionality) so as to store in the $\mathcal{F}_{\mathrm{MPC}}$ functionality the values $\left[n_{i_{j}}\right]=\left[s_{i_{j}}\right]+\left(y_{i_{j}}+r_{i_{j}}\right) \cdot[K]$. The value is then opened to all players by calling (Output, $n_{i_{j}}$ ).

* If $n_{i_{j}} \neq m_{i_{j}}$ then the players call Cheat(1) on the $\mathcal{F}_{\mathrm{MPC}}$ functionality. This will either abort, or return the input of $P_{1}$ (and hence the function), in the latter case the players can now proceed with evaluating the function using standard MPC and without the need for $P_{1}$ to be involved.

\section{- Players Jointly Evaluate Gate $i$.}

* The players store the value $\left[y_{i_{j}}\right]=d_{i_{j}}-\left[r_{i_{j}}\right]$ in the $\mathcal{F}_{\mathrm{MPC}}$ functionality.

* The $\mathcal{F}_{\mathrm{MPC}}$ functionality is then executed so as to compute the output of the gate as

$$
\left[z_{i}\right]=\left(1-\left[G_{i}\right]\right) \cdot\left(\left[y_{i_{0}}\right]+\left[y_{i_{1}}\right]\right)+\left[G_{i}\right] \cdot\left[y_{i_{0}}\right] \cdot\left[y_{i_{1}}\right] .
$$

* Note that the outgoing wire label corresponding to the output wire of the $i$ th gate is $j=n+i$ so we just relabel $\left[z_{i}\right]$ to $\left[z_{j}\right]$.

* If $G_{i}$ is an output gate, players call (Output, $z_{i}$ ) to obtain $z_{i}$, disregard next steps and continue to evaluate next gate.

* The players compute via the MPC functionality $\left[u_{j}\right]=\left[z_{j}\right]+\left[\ell_{j}\right]$.

* The players call (Output, $u_{j}$ ) so as to obtain $u_{j}$.

* The players then compute via the MPC functionality

$$
\left[v_{j}\right]=\left[t_{j}\right]+u_{j} \cdot[K] \doteq\left[t_{j}+\left(z_{j}+\ell_{j}\right) \cdot K\right] .
$$

* The players call $\left(\right.$ Output, $\left.v_{j}\right)$ so as to obtain $v_{j}$.

Fig. 6: The Protocol for implementing PFE 
$\pi$ to values $\left\{\left[\ell_{i}\right]\right\}$ and $\left\{\left[t_{i}\right]\right\}$ to produce shared values $\left\{\left[\ell_{\pi(i)}\right]\right\}$ and $\left\{\left[t_{\pi(i)}\right]\right\}$. In the semi-honest case [17, linear complexity solution for this problem is achieved by employing a singly homomorphic encryption. The shared values are jointly encrypted; $P_{1}$ applies the extended permutation to the resulting ciphertexts and re-randomizes them in order to hide $\pi$; parties jointly decrypt in order to obtain the shares of the resulting plaintexts. To obtain active security, we need to make each step of the following computation actively secure:

1. Players encrypt the shared input (all of which lie in $\mathbf{F}_{p^{k}}$ ) using an encryption scheme, with respect to a public key for which the players can execute a distributed decryption protocol. The resulting ciphertexts are sent to $P_{1}$.

2. Player $P_{1}$ applies the EP and re-randomizes the ciphertexts and sends them back. He then uses the $\mathcal{Z} \mathcal{K}_{\mathrm{EP}}$ protocol to prove his operation has been done correctly.

3. The players then decrypt the permuted ciphertexts and recover shares of the plaintexts.

To implement the first and last steps we use an an instantiation based on ElGamal encryption, see Appendix $\mathrm{A}$. The middle step is more tricky, and we devote the rest of this section to describing this. For the middle step we need a linear zero-knowledge protocol to prove that $P_{1}$ applied a valid EP to the ciphertexts. Proof of a correct shuffle is a well studied problem in the context of Mix-Nets, and linear solutions for it exist [1]. As discussed in Appendix B, however, extending these linear proofs to the case of extended permutations faces some subtle difficulties which we leave as an open question. Instead we aim for a more general construction that uses the currently available proofs of shuffling, in a black-box way.

\subsection{Linear $\mathcal{Z} \mathcal{K}_{\mathrm{EP}}$ Protocol}

After players compute the encryption of the shared inputs, $P_{1}$ knowing the circuit topology, applies the corresponding extended permutation to the ciphertexts. He then re-randomizes the ciphertexts and then "opens" the ciphertexts. Next, we give a linear zero-knowledge protocol $\mathcal{Z} \mathcal{K}_{\mathrm{EP}}$, which enables $P_{1}$ to prove the correctness of his operation (i.e final ciphertexts are the result of $P_{1}$ applying a valid EP to the input ciphertexts). As our first attempt we considered the possibility of extending existing linear proofs of shuffle to get linear proofs of extended permutation. While plausible there are subtle difficulties that need to be addressed. For more details regarding our attempt on extending the method of Furukawa [1110, refer to Appendix $B$. We leave this approach as an open problem. Instead we give a more general construction which makes black-box calls to proof of shuffle. This construction is inspired by the switching network construction of EP given in [17. We first revisit the extended permutation construction of [17].

Assume the EP mapping represented by the function: $\pi:\{1 \ldots n\} \rightarrow\{1 \ldots m\}$ (Which maps $m$ input wires to $n$ output wires $(n \geq m)$ ). Note that in this section we use $n$ and $m$ to denote the size of EP. In a switching network, the number of inputs and outputs are the same, therefore, the construction takes $m$ real inputs of the EP and $n-m$ additional dummy inputs. The construction is divided into three components. Each component takes the output of the previous one as input. Instead of applying the EP in one step, $P_{1}$ applies each component separately and uses a zero-knowledge protocol to prove its correctness. Figure 7 demonstrates the components. Next, we describe each component and identify the required ZK proof.

Table 1 lists the zero-knowledge protocols that we make a black-box use in our $\mathcal{Z} \mathcal{K}_{\mathrm{EP}}$ protocol. Note that we use $P$ and $Q$ for our EC instantiation instead of $g$ and $h$.

- Dummy-value placement component: This takes the real and dummy ciphertexts as input and for each ciphertexts of a real value that is mapped to $k$ different outputs according to $\pi$, outputs the real ciphertexts followed by $k-1$ dummy ciphertexts. This is repeated for each real ciphertext. The resulting output ciphertexts are all re-randomized. The dummy replacement step can be seen as a shuffling of the input ciphertexts. We use a proof of correct shuffle, $\mathcal{Z} \mathcal{K}_{\text {ShUfFLE }}$, for correctness of this component.

- Replication component: This takes the output of the previous component as input. It directly outputs each real ciphertext but replaces each dummy ciphertext with an encryption of the real input that precedes it. At the end of this step, we have the necessary copies for each real input and the dummy inputs are eliminated. Naturally, all the ciphertexts are re-randomized. To prove correctness of this step, 


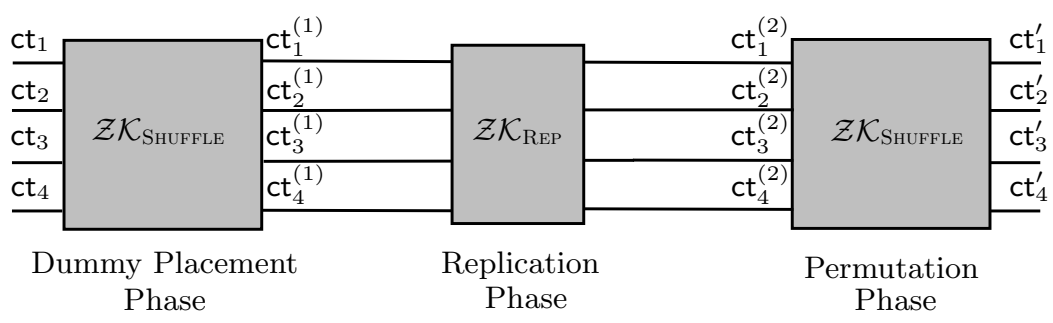

Fig. 7: EP construction. Components' names are written underneath. The zero-knowledge protocol for each component is written inside it's component box.

\begin{tabular}{|c|c|c|}
\hline $\mathcal{Z} \mathcal{K}$ Protocol & Relation/Language & Ref. \\
\hline $\mathcal{Z} \mathcal{K}_{\text {Shuffle }}\left(\left\{\mathfrak{c t}_{i}\right\},\left\{\mathfrak{c t}_{i}^{\prime}\right\}\right)$ & $\begin{aligned} \mathcal{R}_{\text {ShUFfLE }} & =\left\{\left(G, g, h,\left\{\mathfrak{c t}_{i}\right\},\left\{\mathfrak{c t}_{i}^{\prime}\right\}\right) \mid \exists \pi, \text { st. }\right. \\
C_{1}^{\prime(i)} & \left.=g^{r_{i}} C_{1}{ }^{(\pi(i))} \wedge C_{2}^{\prime(i)}=h^{r_{i}} C_{2}{ }^{(\pi(i))} \wedge \pi \text { is perm. }\right\}\end{aligned}$ & 11 \\
\hline $\mathcal{Z} \mathcal{K}_{\mathrm{EQ}_{\mathrm{Q}}}\left(\mathfrak{c t}_{1}, \mathfrak{c t}_{2}\right)$ & $\begin{aligned} \mathcal{R}_{\mathrm{EQ}}=\left\{\left(G, g, h, \mathfrak{c t}_{i}=\right.\right. & \left.\left\langle\alpha_{i}, \beta_{i}\right\rangle_{i \in\{1,2\}}\right) \mid \exists\left(m_{1}, m_{2}\right), \text { st. } \\
& \left.\alpha_{i}=g^{r_{i}} \wedge \beta_{i}=m_{i} h^{r_{i}} \wedge m_{1}=m_{2}\right\}\end{aligned}$ & 5 \\
\hline $\mathcal{Z} \mathcal{K}_{\mathrm{NO}}(\mathfrak{c t})$ & $\begin{array}{r}\mathcal{L}_{\text {NO }}=\left\{(G, g, h, \mathfrak{c t}=\langle\alpha, \beta\rangle) \mid \exists\left(m_{1} \neq 1\right), \text { st. }\right. \\
\left.\alpha=g^{r} \wedge \beta=m_{1} h^{r}\right\}\end{array}$ & 13 \\
\hline
\end{tabular}

Table 1: List of zero-knowledge protocols used in our $\mathcal{Z} \mathcal{K}_{\mathrm{EP}}$ protocol. Generator $g$ and public key $h=g^{s k}$.

we need ZK proofs that the $i$-th output ciphertext has a plaintext equal to that of either the $i$-th input ciphertext or $(i-1)$-th output ciphertext (these can be achieved using protocol $\mathcal{Z} \mathcal{K}_{\mathrm{EQ}}$ defined in Table 1 as a building block). But this is not sufficient to guarantee a correct EP, as we also have to make sure that after the replication component there are no dummy ciphertexts left. For this, we assume that all dummy ciphertexts are encryptions of one. Then for each output ciphertext in the replication component we use a protocol $\mathcal{Z K}_{\mathrm{NO}}$, i.e. a $\mathrm{ZK}$ proof that the underlying plaintext is not one. The $\mathcal{Z} \mathcal{K}_{\mathrm{REP}}$ zero-knowledge protocol, is a compilation of three ZK protocols, two checking for equality of ciphertexts and one checking the inequality of plaintext to one.

- Permutation component: This takes the output of the replication component as input and permutes each element to its final location as prescribed by $\pi$. We again use the proof of correct shuffle, $\mathcal{Z} \mathcal{K}_{\text {ShuFfLE }}$. for this component.

$\mathcal{Z} \mathcal{K}_{\mathrm{EP}}$ Protocol description We assumed the inputs to the $\mathcal{Z} \mathcal{K}_{\mathrm{EP}}$, to be the outputs of our encryption functionality. Prover applies the extended permutation to the ciphertexts $\left(\mathfrak{c t}_{1}, \ldots, \mathfrak{c t}_{n}\right)$, where $\mathfrak{c t}_{i}=\left(C_{1}^{(i)}, C_{2}^{(i)}\right)$. The prover obtains a re-randomized $\left(\mathfrak{c t}_{1}^{\prime}, \ldots, \mathfrak{c t}_{n}^{\prime}\right)$, where $\mathfrak{c t}_{i}^{\prime}=\left(C_{1}^{\prime(i)}, C_{2}^{\prime(i)}\right)$. We employ the techniques of Cramer et al. 6], to combine HVZK proof systems corresponding to each component, at no extra cost, into HVZK proof systems of the same class for any (monotonic) disjunctive and/or conjunctive formula over statements proved in the component proof systems. Figure 8 shows the complete description of our $\mathcal{Z} \mathcal{K}_{\mathrm{EP}}$ protocol. Note that we can choose dummy values from any set of random values $S_{d}$ and substitute the $\mathcal{Z} \mathcal{K}_{\mathrm{NO}}(x)$ with $\vee_{\forall y \in S_{d}}\left(\mathcal{Z} \mathcal{K}_{\mathrm{EQ}}(x, y)\right)$.

Theorem 2. The protocol described in Figure 8 is $H V Z K$ proof of an extended permutation $\pi,\left(\mathfrak{c t}_{1}, \ldots, \mathfrak{c t}_{n}\right)$ and $\left(\mathfrak{c t}_{1}^{\prime}, \ldots, \mathfrak{c t}_{n}^{\prime}\right)$ in the $\mathcal{Z} \mathcal{K}_{\text {ShuffLe }}, \mathcal{Z} \mathcal{K}_{\mathrm{EQ}}, \mathcal{Z K}_{\mathrm{NO}}$ hybrid model, for the following relation:

$$
\mathcal{R}_{\mathrm{EP}}=\left\{\left(G, g, h,\left\{\mathfrak{c t}_{i}\right\},\left\{\mathfrak{c t}_{i}^{\prime}\right\}\right) \mid \exists \pi, \text { st. } C_{1}^{\prime(i)}=g^{r_{i}} C_{1}{ }^{(\pi(i))} \wedge C_{2}^{\prime(i)}=h^{r_{i}} C_{2}{ }^{(\pi(i))} \wedge \pi \text { is } E P .\right\}
$$

Proof. Following is a proof sketch. We show if the construction of EP from [17] is correct, then the $\mathcal{Z} \mathcal{K}_{\mathrm{EP}}$ protocol is a HVZK proof for EP. The goal of first two components is to prepare enough copies of each element. This implies that after the second component no dummy elements should be remained and no new elements are introduced. $\mathcal{Z} \mathcal{K}_{\text {ShUFfLE }}$ and $\mathcal{Z} \mathcal{K}_{\text {ReP }}$ guarantee these two. $\mathcal{Z} \mathcal{K}_{\text {ShUfFle }}$ makes sure no additional 
Shared Input: Ciphertexts $\left(\mathfrak{c t}_{1}, \ldots, \mathfrak{c t}_{n}\right)$

$P_{1}$ 's Input: Extended permutation $\pi$

$P_{1}$ Evaluates the components.

- Player $P_{1}$ finds the corresponding permutation $\pi_{1}$, and $\pi_{2}$ for Dummy-placement component and permutation components.

- $P_{1}$ applies the Dummy-placement component to $\left(\mathfrak{c t}_{1}, \ldots, \mathfrak{c t}_{n}\right)$, and re-randomizes to find $\left(\mathfrak{c t}_{1}^{(1)}, \ldots, \mathfrak{c t}_{n}^{(1)}\right)$.

- $P_{1}$ applies the Replication component to $\left(\mathfrak{c t}_{1}^{(1)}, \ldots, \mathfrak{c t}_{n}^{(1)}\right)$, and re-randomizes them to find $\left(\mathfrak{c t}_{1}^{(2)}, \ldots, \mathfrak{c t}_{n}^{(2)}\right)$.

$-P_{1}$ applies the permutation component to $\left(\mathfrak{c t}_{1}^{(2)}, \ldots, \mathfrak{c t}_{n}^{(2)}\right)$, and re-randomizes them to find $\left(\mathfrak{c t}_{1}^{\prime}, \ldots, \mathfrak{c t}_{n}^{\prime}\right)$.

$P_{1}$ Computes the ZK proofs and sends everything

- Player $P_{1}$ uses the $\mathcal{Z} \mathcal{K}_{\text {Shuffle }}\left(\left\{\mathfrak{c t}_{i}\right\},\left\{\mathfrak{c t}_{i}^{(1)}\right\}\right)$ and $\mathcal{Z} \mathcal{K}_{\text {Shuffle }}\left(\left\{\mathfrak{c t}_{i}^{(2)}\right\},\left\{\mathfrak{c t}_{i}^{\prime}\right\}\right)$ protocols to produce proof of correctness for his evaluation of Dummy-placement component and permutation component.

- Player $P_{1}$ used the $\mathcal{Z} \mathcal{K}_{\mathrm{REP}}\left(\left\{\mathfrak{c t}_{i}^{(1)}\right\},\left\{\mathfrak{c t}_{i}^{(2)}\right\}\right)$ to produce proof of correctness for his evaluation of Replication component as follows(using [6] for combination) (and $\left.\mathcal{Z} \mathcal{K}_{\mathrm{REP}}^{1}=\mathcal{Z} \mathcal{K}_{\mathrm{NO}}\left(\mathfrak{c t}_{1}^{(2)}\right) \wedge \mathcal{Z} \mathcal{K}_{\mathrm{EQ}}\left(\mathfrak{c t}_{1}^{(1)}, \mathfrak{c t}_{1}^{(2)}\right)\right)$ :

- For $2 \leq i \leq n$ :

$$
\mathcal{Z} \mathcal{K}_{\mathrm{REP}}^{i}=\left(\mathcal{Z} \mathcal{K}_{\mathrm{EQ}}\left(\mathfrak{c t}_{i}^{(1)}, \mathfrak{c t}_{i}^{(2)}\right) \vee \mathcal{Z} \mathcal{K}_{\mathrm{EQ}}\left(\mathfrak{c t}_{i-1}^{(2)}, \mathfrak{c t}_{i}^{(2)}\right)\right) \wedge \mathcal{Z} \mathcal{K}_{\mathrm{NO}}\left(\mathfrak{c t}_{i}^{(2)}\right)
$$

- $\mathcal{Z} \mathcal{K}_{\mathrm{REP}}=\wedge_{i=1, \ldots, n}\left(\mathcal{Z} \mathcal{K}_{\mathrm{REP}}^{i}\right)$

- Player $P_{1}$ sends $\left(\mathfrak{c t}_{1}^{(1)}, \ldots, \mathfrak{c t}_{n}^{(1)}\right),\left(\mathfrak{c t}_{1}^{(2)}, \ldots, \mathfrak{c t}_{n}^{(2)}\right),\left(\mathfrak{c t}_{1}^{\prime}, \ldots, \mathfrak{c t}_{n}^{\prime}\right)$ and all proofs to other players.

Players verify $P_{1}$ operations

- Players verify $P_{1}$ 's operations by verifying the the proofs sent by $P_{1}$.

Fig. 8: The protocol for zero-knowledge proof of extended permutation.

elements are introduced in the first component. $\mathcal{Z} \mathcal{K}_{\text {ReP }}$ ensures each element is one of the input pairs to the second component. This makes sure no new elements are introduced in this step. Furthermore, it checks using $\mathcal{Z} \mathcal{K}_{\mathrm{NO}}$ for remaining dummy elements. Note that the EP construction does not require dummy-placement phase to necessarily arrange the elements in any order, and as long as we have satisfied the two mentioned properties, application of any permutation component, results in a valid EP, and also a valid circuit topology. $\mathcal{Z} \mathcal{K}_{\text {Shuffet }}$ is used to check the final component. This sums up the proof. Finally we employ the techniques of Cramer et al. 6], to combine HVZK proof systems corresponding to each component, at no extra cost, into HVZK proof systems of the same class. Note that we make a black-box call to underlying ZK proof systems.

Offline Protocol Having all the parts of the puzzle, we can give the complete $O(g)$ protocol for the offline phase. Figure 9 shows the description, with the proof of security given in Appendix C.

\section{A practical Implementation of $\mathcal{F}_{\text {Offline }}$ with $O(g \cdot \log g)$ Complexity}

A $O(g \cdot \log g)$ protocol to implement $\mathcal{F}_{\text {Offline }}$ is given in Figure 13 and Figure 14 (see Appendix D), and is in the $\mathcal{F}_{\text {MPC}}$-hybrid model. Following the ideas in [17, we implement the functionality via secure evaluation of a switching network corresponding to the mapping $\pi_{f}$.

Switching Networks. A switching network SN is a set of interconnected switches that takes $N$ inputs and a set of selection bits, and outputs $N$ values. Each switch in the network accepts two $\ell$-bit strings as input and outputs two $\ell$-bit strings. In this paper we need to use a switching network that contains two switch types. In the first type (type 1), if the selection bit is 0 the two inputs remain intact and are directly fed to the two outputs, but if the selection bit is 1, the two input values swap places. In the second type (type 2), if the selection bit is 0 , as before, the inputs are directly fed to outputs but if it is 1 , the value of the first input is used for both outputs. For ease of exposition, in our protocol description we assume that all switches are of type 1 , but the protocol can be easily extended to work with both switch types. 


\section{Linear Implementation of Protocol $\mathcal{P}_{\text {OffLine }}$-Linear}

The protocol is described in the $\mathcal{F}_{\mathrm{MPC}}$-hybrid model, thus the only operation we need to specify is the Input

Function one.

Input Function:

$P_{1}$ Shares his Circuit/Function.

- Player $P_{1}$ calls $\left(\right.$ input,$G_{j}$ ) for all $j \in\{1, \ldots, g\}$.

- Players evaluate and open $\left[G_{j}\right] \cdot\left(1-\left[G_{j}\right]\right)$ for $j \in\{1, \ldots, g\}$. If any of them is not 0 , players abort (since in this case $P_{1}$ has not entered a valid function).

Players Generate Randomness for inputs and outputs of EP.

- Players call $($ random,$\cdot)$ of $\mathcal{F}_{\text {MPC }}$ to generate shared random values for inputs $\ell=\left(\left[\ell_{1}\right], \ldots,\left[\ell_{\text {ow }(f)}\right]\right)$ and outputs $\left(\left[r_{1}\right], \ldots,\left[r_{\mathrm{iw}(f)}\right]\right)$ of EP.

- Players call $($ random,$\cdot)$ of $\mathcal{F}_{\mathrm{MPC}}$ to generate shared random values for the MAC value corresponding to inputs $\boldsymbol{t}=\left(\left[t_{1}\right], \ldots,\left[t_{\mathrm{ow}(f)}\right]\right)$ and outputs $\left(\left[s_{1}\right], \ldots,\left[s_{\mathrm{iw}(f)}\right]\right)$ of EP.

$P_{1}$ applies the EP to $\ell$ and $t$.

- The players call KeyGen on the Enc $E_{E l g}$ functionality.

- The playes call Encrypt on the Enc Elg functionality with the plaintexts $\left(\left[\ell_{1}\right], \ldots,\left[\ell_{\mathrm{ow}(f)}\right]\right)$ and the plaintexts $\left(\left[t_{1}\right], \ldots,\left[t_{\mathrm{ow}(f)}\right]\right)$, to obtain ciphertexts $\mathfrak{c t}_{1}, \ldots, \mathfrak{c t}_{\mathrm{ow}(f)}$ and $\mathfrak{c t}_{1}^{\dagger}, \ldots, \mathfrak{c t}_{\mathrm{ow}(f)}^{\dagger}$.

- Player $P_{1}$ applies the extended permutation to $\left(\mathfrak{c t}_{1}, \ldots, \mathfrak{c t}_{\mathrm{ow}(f)}\right)$ and re-randomize to get $\left(\mathfrak{c t}_{1}^{\prime}, \ldots, \mathfrak{c t}_{\mathrm{ow}(f)}^{\prime}\right)$, the same is done with $\left(\mathfrak{c t}_{1}^{\dagger}, \ldots, \mathfrak{c t}_{\mathrm{ow}(f)}^{\dagger}\right)$ to obtain $\left(\mathfrak{c t}_{1}^{\prime \dagger}, \ldots, \mathfrak{c t}_{\mathrm{ow}(f)}^{\prime \dagger}\right)$.

- Player $P_{1}$ uses the $\mathcal{Z} \mathcal{K}_{\mathrm{EP}}$ to prove that he has used a valid extended permutation.

- Players call the Decrypt on the Enc ${ }_{E l g}$ functionality (Figure 11p with ciphertexts $\left(\mathfrak{c t}_{1}^{\prime}, \ldots, \mathfrak{c t}_{\mathrm{ow}(f)}^{\prime}\right)$ and $\left(\mathfrak{c t}_{1}^{\prime \dagger}, \ldots, \mathfrak{c t}_{\mathrm{ow}(f)}^{\prime \dagger}\right)$ so as to obtain $\left(\left[\ell_{\pi(1)}\right], \ldots,\left[\ell_{\pi(\mathrm{ow}(f))}\right]\right)$ and $\left(\left[t_{\pi(1)}\right], \ldots,\left[t_{\pi(\mathrm{ow}(f))}\right]\right)$.

Players Compute $p_{i}, q_{i}$.

- For $i \in\{1, \ldots, \operatorname{iw}(f)\}$ players call $\mathcal{F}_{\text {MPC }}$ to compute:

$$
\left[p_{i}\right]=\left[r_{i}\right]-\left[\ell_{\pi(i)}\right] \doteq\left[r_{i}-\ell_{\pi(i)}\right] \quad, \quad\left[q_{i}\right]=\left[s_{i}\right]-\left[t_{\pi(i)}\right]+p_{i} \cdot[K] \doteq\left[s_{i}-t_{\pi(i)}+p_{i} \cdot K\right]
$$

Fig. 9: The protocol for linear implementation of the Offline Phase 
The mapping $\pi:\{1 \ldots N\} \rightarrow\{1 \ldots N\}$ corresponding to a switching network SN is defined such that $\pi(j)=i$ if and only if after evaluation of SN on the $N$ inputs, the value of the input wire $i$ is assigned to the output wire $j$ (assuming a standard numbering of the input/output wires). In [17] it is shown how to represent any mapping with a maximum of $N$ inputs and outputs via a network with $O(N \cdot \log N)$ type 1 and 2 switches (We refer the reader to [17] for the details). This yields a switching network with $O(g \cdot \log g)$ switches to represent the mapping for a circuit with $g$ gates.

High Level Description. It is possible to implement the $\mathcal{F}_{\text {OfFunE }}$ by securely computing a circuit for the above switching network using the $\mathcal{F}_{\text {MPC }}$. But for all existing MPC that meet our requirements, this would require $O(\log g)$ rounds of interaction which is the depth of the circuit corresponding to the switching network. We show an alternative constant-round approach with similar computation and communication efficiency. It follows the same idea as the OT-based protocol of [17 where the OT is replaced with an equivalent functionality implemented using $\mathcal{F}_{\text {MPC }}$. The main challenge in our case is to achieve active security and in particular to ensure that $P_{1}$ cannot cheat in his local computation. We do so by checking $P_{1}$ 's actions using one-time MACs of the values he computes on, and allow the other parties to learn his input and proceed without him, if he is caught cheating (or aborting).

Next we give an overview of the protocol. The protocol has four main components (as described in Figure 13 and Figure 14). In the first step, $P_{1}$ converts his mapping $\pi$ to selection bits for the switching network (i.e. $\left.b_{i} \mathrm{~s}\right)$ and shares them with all players. He also shares a bit $G_{i}$ indicating the function of gate $i$, with other players. In the second step, players generate random values for every wire in the network. $P_{1}$, based on his selection bit for the switch, learns two of the four possible "subtractions" of the random values for two output wires from those of the input wires i.e. $u_{0}^{\ell, i}$ and $u_{1}^{\ell, i}$. A similar process is performed for the $t$ values to obtain $u_{0}^{t, i}$ and $u_{1}^{t, i}$ (Figure 10 shows this process in a diagram). These subtractions enable $P_{1}$ to transform a pair of values blinded with the random values of input wires, to the same pair of values permuted (based on the selection bit) and blinded with the random values of the output wires. All of the above can be implemented using the operations provided by the $\mathcal{F}_{\text {MPC }}$.

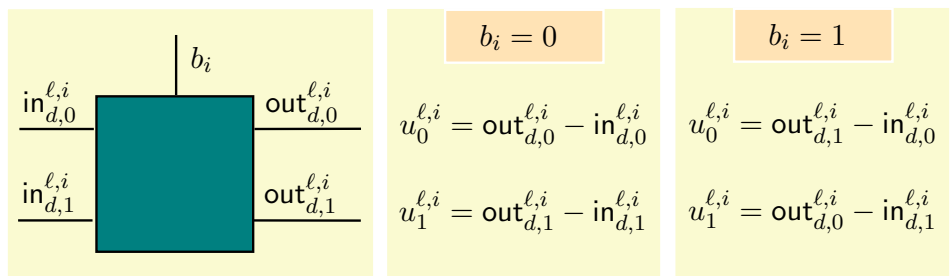

Fig. 10: The $i$-th switch. (superscripts: label of value subject to permute $(\ell$ or $t$ ), and switch index $i$ ) (subscripts: $d$ refers to data, $m$ refers to MAC, wire index 0 denotes the top wire in switch and 1 the bottom wire in switch)

In the third step, $P_{1}$ obtains the blinded $\ell$ and $t$ values where the blinding for each is the random value for the corresponding input wire to the network (these are $h_{d}^{\ell, i}, h_{d}^{t, i}$, etc). Party $P_{1}$ can now process each switch as discussed above using the subtraction values in order to evaluate the entire network. At the end of this process, $P_{1}$ holds blinded values of the outputs of the switching network (blinded with randomness of the output wires).

In the final step, parties check that $P_{1}$ has not cheated during his evaluation, since he performed this step locally and not through the $\mathcal{F}_{\text {MPC }}$ operations. We use one-time MACs to achieve this goal. In particular, besides mapping blinded values through the network, $P_{1}$ also maps the corresponding one-time MACs (generated using the fixed-key $K$ ). This is done using a similar process described above and via the $v_{j}^{\ell, i}, v_{j}^{t, i}$ values. At the end of this process, $P_{1}$ holds one-time MACs for the blinded outputs of the switching network, in addition to the values themselves. Players then use the MPC functionality to jointly verify that the MACs indeed verify the values $P_{1}$ shared with them (i.e. $n^{\ell, i}$ and $m^{\ell, i}$ are the same, etc). As a result, $P_{1}$ can only 
cheat by forging the MACs which only happens with a negligible probability. If the MACs pass, parties compute and open the "difference vectors" by subtracting the mapped $\ell$ and $t$-value vectors from the $r$ and $s$-value vectors. Refer to Figure 13 and Figure 14 for more details. If one instantiates the $\mathcal{F}_{\text {MPC }}$ by SPDZ [8], which has the $m \cdot \log \left(p^{k}\right)$ complexity, then our complexity would be $m(10(2 g \log 2 g-2 g+1)+4 g) \cdot \log \left(p^{k}\right)$. Refer to Appendix Efor the proof of the following theorem.

Theorem 3. In the $\mathcal{F}_{\mathrm{MPC}}$-hybrid model the protocol $\mathcal{P}_{\text {OFFLINE }}$ in Figure 13 and Figure 14 securely implements the functionality in Figure 2, with complexity $O(g \cdot \log g)$.

\section{Acknowledgements}

This work has been supported in part by ERC Advanced Grant ERC-2010-AdG-267188-CRIPTO, by EPSRC via grant EP/I03126X, and by Defense Advanced Research Projects Agency (DARPA) and the Air Force Research Laboratory (AFRL) under agreement number FA8750-11-2-0079.

The US Government is authorized to reproduce and distribute reprints for Government purposes notwithstanding any copyright notation thereon. The views and conclusions contained herein are those of the authors and should not be interpreted as necessarily representing the official policies or endorsements, either expressed or implied, of Defense Advanced Research Projects Agency (DARPA) or the U.S. Government.

\section{References}

1. M. Abadi and J. Feigenbaum. Secure circuit evaluation. J. Cryptology, 2(1):1-12, 1990.

2. M. Barni, P. Failla, V. Kolesnikov, R. Lazzeretti, A.-R. Sadeghi, and T. Schneider. Secure evaluation of private linear branching programs with medical applications. In M. Backes and P. Ning, editors, ESORICS, volume 5789 of Lecture Notes in Computer Science, pages 424-439. Springer, 2009.

3. R. Bendlin, I. Damgård, C. Orlandi, and S. Zakarias. Semi-homomorphic encryption and multiparty computation. In K. G. Paterson, editor, EUROCRYPT, volume 6632 of Lecture Notes in Computer Science, pages 169-188. Springer, 2011.

4. J. Brickell, D. E. Porter, V. Shmatikov, and E. Witchel. Privacy-preserving remote diagnostics. In P. Ning, S. D. C. di Vimercati, and P. F. Syverson, editors, ACM Conference on Computer and Communications Security, pages 498-507. ACM, 2007.

5. D. Chaum and T. P. Pedersen. Wallet databases with observers. In CRYPTO, pages 89-105, 1992.

6. R. Cramer, I. Damgård, and B. Schoenmakers. Proofs of partial knowledge and simplified design of witness hiding protocols. In Y. Desmedt, editor, Advances in Cryptology - CRYPTO '94, volume 839 of Lecture Notes in Computer Science, pages 174-187. Springer Berlin Heidelberg, 1994.

7. I. Damgård, M. Geisler, M. Krøigaard, and J. B. Nielsen. Asynchronous multiparty computation: Theory and implementation. In Proceedings of the 12th International Conference on Practice and Theory in Public Key Cryptography: PKC '09, Irvine, pages 160-179, Berlin, Heidelberg, 2009. Springer-Verlag.

8. I. Damgård, V. Pastro, N. P. Smart, and S. Zakarias. Multiparty computation from somewhat homomorphic encryption. In Safavi-Naini and Canetti [25], pages 643-662.

9. I. Damgård and S. Zakarias. Constant-overhead secure computation of boolean circuits using preprocessing. In Theory of Cryptography, pages 621-641. Springer, 2013.

10. J. Furukawa. Efficient and verifiable shuffling and shuffle-decryption. IEICE Transactions, 88-A(1):172-188, 2005.

11. J. Furukawa and K. Sako. An efficient scheme for proving a shuffle. In J. Kilian, editor, Advances in Cryptology CRYPTO 2001, volume 2139 of Lecture Notes in Computer Science, pages 368-387. Springer Berlin Heidelberg, 2001.

12. R. Gennaro, C. Hazay, and J. S. Sorensen. Text search protocols with simulation based security. In P. Q. Nguyen and D. Pointcheval, editors, Public Key Cryptography, volume 6056 of Lecture Notes in Computer Science, pages 332-350. Springer, 2010.

13. C. Hazay and K. Nissim. Efficient set operations in the presence of malicious adversaries. In Public Key Cryptography, pages 312-331, 2010.

14. Y. Ishai and A. Paskin. Evaluating branching programs on encrypted data. In S. P. Vadhan, editor, TCC, volume 4392 of Lecture Notes in Computer Science, pages 575-594. Springer, 2007. 
15. J. Katz and L. Malka. Constant-round private function evaluation with linear complexity. In D. H. Lee and X. Wang, editors, ASIACRYPT, volume 7073 of Lecture Notes in Computer Science, pages 556-571. Springer, 2011.

16. V. Kolesnikov and T. Schneider. A practical universal circuit construction and secure evaluation of private functions. In G. Tsudik, editor, Financial Cryptography, volume 5143 of Lecture Notes in Computer Science, pages 83-97. Springer, 2008.

17. P. Mohassel and S. Sadeghian. How to hide circuits in MPC an efficient framework for private function evaluation. In T. Johansson and P. Q. Nguyen, editors, EUROCRYPT, volume 7881 of Lecture Notes in Computer Science, pages 557-574. Springer, 2013.

18. C. A. Neff. A verifiable secret shuffle and its application to e-voting. In M. K. Reiter and P. Samarati, editors, ACM Conference on Computer and Communications Security, pages 116-125. ACM, 2001.

19. J. B. Nielsen, P. S. Nordholt, C. Orlandi, and S. S. Burra. A new approach to practical active-secure two-party computation. In Safavi-Naini and Canetti [25], pages 681-700.

20. S. Niksefat, B. Sadeghiyan, P. Mohassel, and S. Sadeghian. Zids: A privacy-preserving intrusion detection system using secure two-party computation protocols. The Computer Journal, 2013.

21. R. Ostrovsky, A. Paskin-Cherniavsky, and B. Paskin-Cherniavsky. Maliciously circuit-private fhe. Cryptology ePrint Archive, Report 2013/307, 2013. http://eprint.iacr.org/

22. A. Paus, A.-R. Sadeghi, and T. Schneider. Practical secure evaluation of semi-private functions. In M. Abdalla, D. Pointcheval, P.-A. Fouque, and D. Vergnaud, editors, ACNS, volume 5536 of Lecture Notes in Computer Science, pages 89-106, 2009.

23. R. Raz. Elusive functions and lower bounds for arithmetic circuits. In Proceedings of the Fortieth Annual ACM Symposium on Theory of Computing, STOC '08, pages 711-720, New York, NY, USA, 2008. ACM.

24. A.-R. Sadeghi and T. Schneider. Generalized universal circuits for secure evaluation of private functions with application to data classification. In P. J. Lee and J. H. Cheon, editors, ICISC, volume 5461 of Lecture Notes in Computer Science, pages 336-353. Springer, 2008.

25. R. Safavi-Naini and R. Canetti, editors. Advances in Cryptology - CRYPTO 2012 - 32nd Annual Cryptology Conference, Santa Barbara, CA, USA, August 19-23, 2012. Proceedings, volume 7417 of Lecture Notes in Computer Science. Springer, 2012.

26. L. Valiant. Universal circuits (preliminary report). In Proceedings of the eighth annual ACM symposium on Theory of computing, pages 196-203. ACM, 1976.

\section{A Instantiating Shared Encryption/Decryption}

Recall our messages are elements in $\mathbf{F}_{p^{k}}$ and we aim to work in an elliptic curve group of prime order (to ensure DDH holds in the whole group). We therefore consider the finite field $\mathbf{F}_{p^{2 k}}=\mathbf{F}_{p^{k}}[\theta]$, and consider an elliptic curve $E\left(\mathbf{F}_{p^{2 k}}\right)$ of prime order $q$ with generator $P$. Let the curve be given by the equation $Y^{2}=X^{3}+A \cdot X+B$ where $A, B \in \mathbf{F}_{p^{2 k}}$. To encrypt an element $m \in \mathbf{F}_{p^{k}}$ we map elements of $\mathbf{F}_{p^{k}}$ to elliptic curve points as follows: We pick a random $r \in \mathbf{F}_{p^{k}}$ and set $x=m+r \cdot \theta$. If $t=x^{3}+A \cdot x+B$ is a square (which can be tested by checking if $t^{\left(p^{2 k}-1\right) / 2}=1$ ), we extract the square root $y$ (by the Tonelli-Shanks algorithm) and return $M=(x, y)$, otherwise we pick another $r$ and repeat the operation. We expect this process to terminate after two steps on average.

Given $M$ we can encrypt it by selecting $k \in \mathcal{Z}_{q}$ and computing $\left(C_{1}, C_{2}\right)=(k \cdot P, M+k \cdot Q)$ where $Q=\mathfrak{s k} \cdot P$ is the public key corresponding to the secret key $\mathfrak{s k}$. The decryption can be obtained via $C_{2}-\mathfrak{s k} \cdot C_{1}$, and then simply taking the $x$-coordinate as $x_{0}+x_{1} \cdot \theta$ and returning $x_{0}$.

We need to perform the encryption however on values which are shared via the $\mathcal{F}_{\text {MPC }}$ functionality, and decrypt to obtain values which are shared via the $\mathcal{F}_{\text {MPC }}$ functionality. We first note that since the $\mathcal{F}_{\text {MPC }}$ functionality can evaluate arithmetic circuits over $\mathbf{F}_{p^{k}}$ it can also evaluate circuits over $\mathbf{F}_{p^{2 k}}$; so for ease of exposition we will assume that $\mathcal{F}_{\mathrm{MPC}}$ is defined over $\mathbf{F}_{p^{2 k}}$. We can therefore define the functionality Enc Elg given in Figure 11 in the $\mathcal{F}_{\mathrm{MPC}}$-hybrid model. To ease notation we let $[P]$ denote a sharing of an elliptic curve point $P$ in the $\mathcal{F}_{\mathrm{MPC}}$ functionality in what follows. To save space we have included the protocol to implement $\mathcal{F}_{\text {MPC }}$ within the description of the functionality itself. 


\section{Functionality Enc $E l g$}

KeyGen: This generates the public key for the ElGamal encryption, given a shared secret key. The secret key is stored as shared bits for convenience, i.e. $[\mathfrak{s k}]=\sum\left[\mathfrak{s k}_{i}\right] \cdot 2^{i}$.

1. Player $i$ calls $\left(\right.$ input $\left., P_{i}, \mathfrak{s k}_{i, j}, x_{i, j}\right)$ for $j=0, \ldots, \log _{2} q$ and randomly selected $x_{i, j} \in\{0,1\}$ chosen by player $i$.

2. Define $[Q]$ as the sharing of the point at infinity.

3. This step forms $\left[\mathfrak{s k}_{i}\right]=\bigoplus\left[\mathfrak{s k}_{j, i}\right]$ and $[Q]=\sum\left[\mathfrak{s k}_{i}\right] \cdot 2^{i} \cdot P$, and ensures that the players input values in the first step are in $\{0,1\}$. We perform this step by executing, for $i=0, \ldots, \log _{2} q$,

$-\left[\mathfrak{s k}_{i}\right]=\left[\mathfrak{s k}_{0, i}\right]$

- For $j=2, \ldots, n$ do $\left[\mathfrak{s k}_{i}\right]=-2 \cdot\left[\mathfrak{s k}_{i}\right] \cdot\left[\mathfrak{s k}_{j, i}\right]+\left[\mathfrak{s k}_{i}\right]+\left[\mathfrak{s k}_{j, i}\right]$, using the MPC functionality.

- Compute $\left[t_{i}\right]=\left[\mathfrak{s k}_{i}\right] \cdot\left(\left[\mathfrak{s k}_{i}\right]-1\right)$, again using the MPC functionality.

- Call (output, $t_{i}$ ) to open $\left[t_{i}\right]$, if the value is not zero then restart.

- Execute $[Q]=[Q]+\left[\mathfrak{s k}_{i}\right] \cdot 2^{i} \cdot P$. Here we use the $\mathcal{F}_{\mathrm{MPC}}$ functionality to evaluate the conditional elliptic curve addition.

4. The players call (output, $Q$ ) to open $[Q]$.

Encrypt: This takes an input message $[m]$ where $m \in \mathbf{F}_{p^{k}}$ and outputs an ElGamal ciphertext $\left(C_{1}, C_{2}\right)$.

1. Using a method similar to that for KeyGen above the players generate sharings of bits $\left[k_{i}\right]$ for $i=$ $0, \ldots, \log _{2} q$ and then evaluate $[k P]$ and $[k Q]$ for $k$ the integer with bit representation given by the shared bits $\left[k_{i}\right]$.

2. The players call (random, $r$ ).

3. The players execute $[x]=[m]+\theta \cdot[r]$.

4. The players execute $[t]=\left[x^{3}\right]+A \cdot[x]+B$.

5. The players compute $[s]=\left[t^{\left(p^{2 k}-1\right) / 2}\right]$ and call (output, $s$ ) to open $[s]$.

6 . If $s \neq 1$ then goto step 2

7. The players execute the Tonelli-Shanks algorithm to extract the square root $[y]$ of $[t]$ using the $\mathcal{F}_{\mathrm{MPC}}$ functionality.

8. The players execute $[G]=([x],[y])+[k Q]$.

9. The players call (output, $\cdot$ ) on the $x$ and $y$ coordinates of $[k P]$ and $[G]$ so as to obtain $C_{1}$ and $C_{2}$.

Decrypt: Obtain the sharing of the message $[m]$ corresponding to ciphertext $\left(C_{1}, C_{2}\right)$.

1. The players execute using $\mathcal{F}_{\text {MPC }}$ the operations corresponding to $[G]=C_{2}-\sum_{i=0}^{\log _{2} q}\left[\mathfrak{s k}_{i}\right] \cdot 2^{i} \cdot C_{1}$.

2. Consider $[G]$ as having $x$-coordinate $[m]+\theta \cdot\left[m^{\prime}\right]$ and output $[m]$.

Fig. 11: Elgamal Functionality 


\section{B An Incomplete Attempt to Extend Existing Proofs of Shuffle}

In this section we explain our attempt at extending the existing proofs of shuffle to extended permutation. Current available solutions are following two main ideas: The first group started by Furukawa and Sako [1] represents the permutation by a permutation matrix and then proves using ZK that it is a valid permutation and is used in computation. The second group started by Neff [18] uses the property of polynomials of being identical under permutation of their roots.

In the second group, it is not obvious how it is possible to handle variant number of repetitions for each root. On the other hand it is possible to represent an EP using a matrix.

We turn to modifying the method of Furukawa and Sako [11] (and the later work by Furukawa [10]), to check an extended permutation. We only describe the general idea, for more details concerning our modifications we refer the reader to the original paper [11]. In their protocol they use the matrix representation of permutation and prove that the matrix used for computation of outputs is a valid permutation (i.e. there is exactly one non-zero element one in each row and each column). For our purpose of extended permutation, it is only enough to show that there is exactly one non-zero element, one in each column of matrix. Theorem 4 shows the conditions for a matrix to be an extended permutation.

Theorem 4. A matrix $\left(A_{i j}\right)_{i, j=1, \ldots, n)}$ is an extended permutation if and only if, for all $i, j$ and $k$, the following conditions hold:

$$
\sum_{h=1}^{n} A_{h i}=1 \quad(\bmod q)
$$

For all $i, j:(i \neq j)$

$$
\sum_{h=1}^{n} A_{i h} \cdot A_{j h}=0 \quad(\bmod q)
$$

For all $i, j, k: \neg(i=j=k)$

$$
\sum_{h=1}^{n} A_{i h} \cdot A_{j h} \cdot A_{k h}=0 \quad(\bmod q)
$$

Proof (sketch). The first condition implies that there is at least one non-zero element in each column. Using the similar argument to [11, for $i \neq j$, the second and third conditions imply that the number of non-zero elements in each column is at most one. From first condition, this non-zero element should be one.

This theorem allows us to adapt the zero-knowledge protocol given in [11. The main challenge in their protocol is to give proof for the conditions of equations 223. We assume that the prover has applied the extended permutation to the ciphertexts $\left(\mathfrak{c t}_{1}, \ldots, \mathfrak{c t}_{n}\right)$, where $\mathfrak{c t}_{i}=\left(C_{1}^{(i)}, C_{2}^{(i)}\right)$. The prover obtains a rerandomized $\left(\mathfrak{c t}_{1}^{\prime}, \ldots, \mathfrak{c t}_{n}^{\prime}\right)$, where $\mathfrak{c t}_{i}^{\prime}=\left(C_{1}^{\prime(i)}, C_{2}^{\prime(i)}\right)$ and $C_{1}^{\prime(i)}=k_{i}^{\prime} \cdot P+C_{1}^{(\pi(i))}, C_{2}^{\prime(i)}=k_{i}^{\prime} \cdot Q+C_{2}^{(\pi(i))}$.

To prove the condition in equation 2 , we have to show that given $\left\{C_{1}^{(i)}\right\}$ and $\left\{C_{1}^{\prime(i)}\right\}$, the prover knows $k_{i}^{\prime}$ and $A_{i j}$ such that:

$$
C_{1}^{(i)}=k_{i}^{\prime} \cdot P+\sum_{j=0}^{n} A_{i j} \cdot C_{1}^{(j)} \quad \text { and } \quad \sum_{h=1}^{n} A_{i h} \cdot A_{j h}=0 .
$$

In 11] they suggest to issue values $s$ and $s_{i}$ as a respond to challenge $c_{j}$ and let the verifier check two conditions. We adjust $s_{i}$ for our modified scenario such that $s_{i}^{2}$ generates the condition of equation 2 .

$$
s_{i}=\sum_{j=1}^{n} A_{j i} c_{j} \quad(\bmod q)
$$

At this point it is not obvious how to issue $s$, and define the second verification equation considering the modified $s_{i}$. 


\section{Proof of Protocol $\mathcal{P}_{\text {Offline-Linear }}$}

We construct a simulator $\mathcal{S}_{\text {OffLINE }}$ such that a poly-time environment $\mathcal{Z}$ cannot distinguish between the real protocol system and the ideal. We assume here static, active corruption. The simulator runs a copy of the protocol given in Figure 9, which simulates the ideal functionality given in Figure 2, It relays messages between parties $/ \mathcal{F}_{\text {MPC }}$ and $\mathcal{Z}$, such that $\mathcal{Z}$ will see the same interface as when interacting with a real protocol. The specification of the simulator $\mathcal{S}_{\text {OFFLINE }}$ is presented in Figure 12 .

Simulator $\mathcal{S}_{\text {OFFLINE }}$

The protocol is described in the $\mathcal{F}_{\mathrm{MPC}}$-hybrid model, thus we only need to specify the simulator for the Input Function one. Let's denote the set of corrupted parties by $C \subset\left\{P_{1}, \ldots, P_{N}\right\}$.

\section{Input Function:}

$P_{1}$ Shares his Circuit/Function.

$-P_{1} \in C$ :

- Simulator $\mathcal{S}_{\text {OfFLINE }}$ evaluates $\left[G_{j}\right] \cdot\left(1-\left[G_{j}\right]\right)$ for $j \in\{1, \ldots, g\}$. If any of them is not 0 , simulator abort (since in this case $P_{1}$ has not entered a valid function).

$-P_{1} \notin C$ :

- Simulator $\mathcal{S}_{\text {Offline }}$ generates a random circuit with $g$ gates $G_{j}^{\prime}$ for all $j \in\{1, \ldots, g\}$ and finds its corresponding mapping $\pi^{\prime}$.

- $\mathcal{S}_{\text {OffLine }}$ calls $\left(\right.$ input,$G_{j}^{\prime}$ ) for all $j \in\{1, \ldots, g\}$.

- Simulator $\mathcal{S}_{\text {OfFLINE }}$ evaluates $\left[G_{j}^{\prime}\right] \cdot\left(1-\left[G_{j}^{\prime}\right]\right)$ for $j \in\{1, \ldots, g\}$.

Players Generate Randomness for inputs and outputs of EP.

- Simulator $\mathcal{S}_{\text {OfFune }}$ follows the protocol honestly.

$P_{1}$ Applies the EP to $s \ell$ and $s t$.

$-P_{1} \in C$ :

- Simulator $\mathcal{S}_{\text {OffLINE }}$ randomly generates an extended permutation $\pi^{\prime}$ and sends it to $\mathcal{Z} \mathcal{K}_{\text {EP }}$ ideal functionality. Simulator aborts if any of players aborts.

$-P_{1} \notin C:$

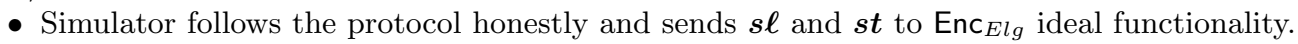

- Simulator $\mathcal{S}_{\text {OfFLINE }}$ waits for $P_{1}$ to broadcast $\pi$ to $\mathcal{Z} \mathcal{K}_{\mathrm{EP}}$ ideal functionality, he then sends $\pi$ to ideal functionality $\mathcal{F}_{\text {Offline }}$. Simulator aborts if any of players aborts.

- Simulator follows the protocol honestly.

Players Check $P_{1}$ 's Work and Compute $p_{i}, q_{i}$.

- Simulator $\mathcal{S}_{\text {OfFLINE }}$ follows the protocol honestly.

Fig. 12: Simulator $\mathcal{S}_{\text {OffLINE }}$

To see that the simulated and real processes cannot be distinguished, we will show that the view of the environment in the ideal process is statistically indistinguishable from the view in the real process. This view consists of the corrupt players' view of the protocol execution as well as the inputs and outputs of honest players.

The view of adversaries $C-\left\{P_{1}\right\}$, includes the share of $G_{i}$, the share of random values for inputs and outputs of EP, $\left(\left[s \ell_{1}\right], \ldots,\left[s \ell_{\mathrm{ow}(f)}\right]\right),\left(\left[s r_{1}\right], \ldots,\left[s r_{\mathrm{iw}(f)}\right]\right),\left(\left[s t_{1}\right], \ldots,\left[s t_{\mathrm{ow}(f)}\right]\right),\left(\left[s s_{1}\right], \ldots,\left[s s_{\mathrm{iw}(f)}\right]\right),\left(\left[s \ell_{\pi(1)}\right], \ldots,\left[s \ell_{\pi(\mathrm{ow}(f))}\right]\right)$,

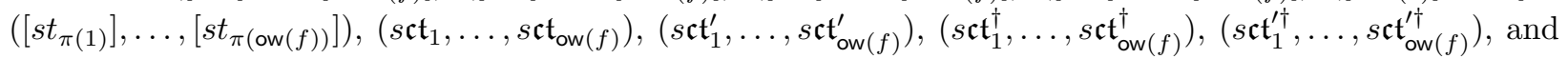
finally, $p_{i}, q_{i}$. The shared values all look random and therefore are indistinguishable between ideal and real execution. $\left(s \mathfrak{c t}_{1}, \ldots, s \mathrm{ct}_{\mathrm{ow}(f)}\right)$ and $\left(\boldsymbol{s c t}_{1}^{\dagger}, \ldots, s \mathfrak{c t}_{\mathrm{ow}(f)}^{\dagger}\right)$ are ElGamal encryptions under shared secret key, and therefore are indistinguishable from real execution. $\left(s \mathfrak{c t}_{1}^{\prime}, \ldots, s \mathfrak{c t}_{\mathrm{ow}(f)}^{\prime}\right)$ and $\left(s \mathfrak{c t}_{1}^{\prime \dagger}, \ldots, s \mathfrak{c t}_{\mathrm{ow}(f)}^{\prime \dagger}\right)$ are valid rerandomization of ElGamal ciphertexts if protocol does not abort due to $\mathcal{Z} \mathcal{K}_{\mathrm{EP}}$ verification. $\left(\left[s \ell_{\pi(1)}\right], \ldots,\left[s \ell_{\pi(\text { ow }(f))}\right]\right)$, $\left(\left[s t_{\pi(1)}\right], \ldots,\left[s t_{\pi(\mathrm{ow}(f))}\right]\right)$ are freshly new shares generated by $\mathrm{Enc}_{E l g}$ protocol. The final result $p_{i}, q_{i}$ is computed as a result of two shared random values, and therefore has a uniform distribution in both ideal and 
real executions. The view of malicious $P_{1}$, is the same view as other malicious players. The shared values all have uniform distribution. In the ideal functionality we also have a uniform distribution, and as a result ideal and real executions are indistinguishable to the environment $\mathcal{Z}$.

\section{Complete Description of Protocol $\mathcal{P}_{\text {Offline }}$}

See Figure 13 and Figure 14 for the description of protocol $\mathcal{P}_{\text {OfflinE }}$.

\section{E Proof of Theorem 3}

We construct a simulator $\mathcal{S}_{\text {OfFLINE }}$ such that a poly-time environment $\mathcal{Z}$ cannot distinguish between the real protocol system and the ideal. We assume here static, active corruption. The simulator runs a copy of the protocol given in Figure 13 and Figure 14, which simulates the ideal functionality given in Figure 2, It relays messages between parties $/ \mathcal{F}_{\text {MPC }}$ and $\mathcal{Z}$, such that $\mathcal{Z}$ will see the same interface as when interacting with a real protocol. The specification of the simulator $\mathcal{S}_{\text {OFFLINE }}$ is presented in Figure 15

To see that the simulated and real processes cannot be distinguished, we will show that the view of the environment in the ideal process is statistically indistinguishable from the view in the real process. This view consists of the corrupt players' view of the protocol execution as well as the inputs and outputs of honest players.

The view of adversaries $C-\left\{P_{1}\right\}$, includes the share of $b_{i}, G_{i}$, the share of wires' random values, $h_{d}^{\ell, i}, h_{d}^{t, i}$, $\left[d^{\ell, i}\right],\left[d^{t, i}\right]$ and finally, $n^{\ell, i}$ and $p_{i}, q_{i}$. The shared values all look random and therefore are indistinguishable between ideal and real execution. The final result $p_{i}, q_{i}$ is computed as a result of two shared random values, and therefore has a uniform distribution in both ideal and real execution. The values $h_{d}^{\ell, i}, h_{d}^{t, i}$ are blinded by shared values $\ell$ and $t$ respectively and have uniform distribution.

The view of malicious $P_{1}$, includes the share of $b_{i}, G_{i}$, share of wires' random values, $h_{d}^{\ell, i}, h_{d}^{t, i}, d^{\ell, i}, d^{t, i}$ and finally, $n^{\ell, i}$ and $p_{i}, q_{i}$. $P_{1}$ has the same view as other malicious players except for the $d^{\ell, i}, d^{t, i}$ values that he has computed. It only remains to show that $d^{\ell, i}, d^{t, i}$ have uniform distribution for a malicious $P_{1}$ and checks are guaranteeing the correctness of his computation. Observe that $h_{d}^{\ell, i}$ is blinded using random value of input wires which is shared and therefore acts as a one-time pad, and as $P_{1}$ does the evaluation the distribution remains uniform as he continues. Using the similar argument, $d^{t, i}$ has a uniform distribution due to $h_{d}^{t, i}$. In the ideal functionality we also have a uniform distribution, and as a result ideal and real are indistinguishable to the environment $\mathcal{Z}$. In the final phase players check the $P_{1}$ 's computation. Player $P_{1}$ cheating means he has not calculated $d^{\ell, i}, d^{t, i}$ correctly. For him to be successful, he has to somehow adjust $n^{\ell, i}$ and $m^{\ell, i}$ to be equal. Any modification is prevented by the fact that since he does not know the key $K$, it acts as a one-time MAC and therefore he can not adjust his share [out $\left.{ }_{m, j}^{\ell,[i / 2\rceil}\right]$ to make the equality hold. The probability of him getting away with it is equal to him guessing $K$ and hence exponentially small in the length of $K$. It follows that with overwhelming probability after the check $P_{1}$ 's computation has been done correctly. If any check fails the simulator aborts and stop.

\section{F Proof of Protocol $\mathcal{P}_{\text {Online }}$}

We construct a simulator $\mathcal{S}_{\text {ONLINE }}$ such that a poly-time environment $\mathcal{Z}$ cannot distinguish between the real protocol system and the ideal. We assume here static, active corruption. The simulator runs a copy of the protocol $\mathcal{P}_{\text {ONLINE }}$ given in Figure 6, which simulates the ideal functionalities given in Figure 44 It relays messages between parties $/ \mathcal{F}_{\text {OFFLINE }}$ and $\mathcal{Z}$, such that $\mathcal{Z}$ will see the same interface as when interacting with a real protocol. The specification of the simulator $\mathcal{S}_{\text {ONLINE }}$ is presented in Figure 16. 
Protocol $\mathcal{P}_{\text {Offline }}$ Part I

The protocol is described in the $\mathcal{F}_{\mathrm{MPC}}$-hybrid model, thus the only operation we need to specify is the Input Function one.

\section{Input Function:}

$P_{1}$ Shares his Circuit/Function.

- $P_{1}$ determines a vector of selection bits $\left(b_{1}, \ldots, b_{N}\right)$ corresponding to the switching network representing the mapping $\pi$. Note that the switching network has ow $(f)$ input wires and iw $(f)$ output wires.

- Player $P_{1}$ calls $\left(\right.$ input,$\left.b_{i}\right)$ for all $i \in\{1, \ldots, N\}$.

- Player $P_{1}$ calls $\left(\right.$ input,$G_{j}$ ) for all $j \in\{1, \ldots, g\}$.

- Players evaluate and open $\left[b_{i}\right] \cdot\left(1-\left[b_{i}\right]\right)$ for all $i \in\{1, \ldots, N\}$ and similarly for $\left[G_{j}\right] \cdot\left(1-\left[G_{j}\right]\right)$ for $j \in\{1, \ldots, g\}$. If any of them is not 0 , players abort (since in this case $P_{1}$ has not entered a valid function).

Players Generate Randomness for the Switching Network.

- The players call (random, $K$ ) of $\mathcal{F}_{\text {MPC }}$.

- Players call $($ random,$\cdot)$ of $\mathcal{F}_{\mathrm{MPC}}$ to generate two pairs of shared random values for each wire in the switching network; one pair is used to map the $\ell$ values and another to map the $t$ values (recall each value $j \in\{1, \ldots$, ow $(f)\}$ has a value $\ell_{j}$ and $\left.t_{j}\right)$.

Let us denote the two shared random pairs for the $j$ th input wire $(j \in\{0,1\})$ of the $i$ th switch by $\left(\left[\operatorname{in}_{d, j}^{\ell, i}\right],\left[\operatorname{in}_{m, j}^{\ell, i}\right]\right)$ and $\left(\left[\operatorname{in}_{d, j}^{t, i}\right],\left[\mathrm{in}_{m, j}^{t, i}\right]\right)$, and the pairs for its two output wires by $\left(\left[\right.\right.$ out $\left._{d, j}^{\ell, i}\right],\left[\right.$ out $\left.\left._{m, j}^{\ell, i}\right]\right)$ and ([out $\left.{ }_{m, i}^{t, i}\right],\left[\right.$ out $\left.\left._{m, j}^{t, i}\right]\right)$. (The $d$ subscript means the random value is used to process data (actual wire values) while the $m$ subscripts means the random value is used for the corresponding macs. The subscript $j \in\{0,1\}$ determines which wire of the switch the value corresponds to. 0 means the the top wire while 1 denotes the bottom wire.)

- Then, for each switch $i$ in the network players perform the following (in parallel):

- The players call $\mathcal{F}_{\mathrm{MPC}}$ to evaluate and open the following for $j \in\{0,1\}$ (the following corresponds to switch type 1 but a similar approach works for type 2 switches)

$$
\begin{aligned}
{\left[u_{j}^{\ell, i}\right]=} & \left(1-\left[b_{i}\right]\right) \cdot\left(\left[\text { out }_{d, j}^{\ell, i}\right]-\left[\text { in }_{d, j}^{\ell, i}\right]\right)+\left[b_{i}\right] \cdot\left(\left[\text { out }_{d, 1-j}^{\ell, i}\right]-\left[\text { in }_{d, j}^{\ell, i}\right]\right), \\
{\left[u_{j}^{t, i}\right]=} & \left(1-\left[b_{i}\right]\right) \cdot\left(\left[\text { out }_{d, j}^{t, i}\right]-\left[\text { in }_{d, j}^{t, i}\right]\right)+\left[b_{i}\right] \cdot\left(\left[\text { out }_{d, 1-j}^{t, i}\right]-\left[\text { in }_{d, j}^{t, i}\right]\right), \\
{\left[v_{j}^{\ell, i}\right]=} & \left(1-\left[b_{i}\right]\right) \cdot\left(\left[\text { out }_{m, j}^{\ell, i}\right]-\left[\mathrm{in}_{m, j}^{\ell, i}\right]\right)+\left[b_{i}\right] \cdot\left(\left[\text { out }_{m, 1-j}^{\ell, i}\right]-\left[\mathrm{in}_{m, j}^{\ell, i}\right]\right) \\
& +u_{j}^{\ell, i} \cdot[K], \\
{\left[v_{j}^{t, i}\right]=} & \left(1-\left[b_{i}\right]\right) \cdot\left(\left[\text { out }_{m, j}^{t, i}\right]-\left[\text { in }_{m, j}^{t, i}\right]\right)+\left[b_{i}\right] \cdot\left(\left[\text { out }_{m, 1-j}^{t, i}\right]-\left[\text { in }_{m, j}^{t, i}\right]\right) \\
& +u_{j}^{t, i} \cdot[K] .
\end{aligned}
$$

Note, the final two equations can be evaluated using the open values of $u_{j}^{\ell, i}$ and $u_{j}^{t, i}$.

- For $i \in\{1, \ldots$, ow $(f)\}$ players call $\mathcal{F}_{\text {MPC }}$ to evaluate and open (let $\left.j=i \bmod 2\right)$

$$
\begin{array}{ll}
{\left[h_{d}^{\ell, i}\right]=\left[\ell_{i}\right]+\left[\operatorname{in}_{d, j}^{\ell,\lceil i / 2\rceil}\right],} & {\left[h_{m}^{\ell, i}\right]=\left[\operatorname{in}_{m, j}^{\ell,\lceil i / 2\rceil}\right]+h_{d}^{\ell, i} \cdot[K],} \\
{\left[h_{d}^{t, i}\right]=\left[t_{i}\right]+\left[\operatorname{in}_{d, j}^{t,\lceil i / 2\rceil}\right],} & {\left[h_{m}^{t, i}\right]=\left[\operatorname{in}_{m, j}^{t,\lceil i / 2\rceil}\right]+h_{d}^{t, i} \cdot[K],}
\end{array}
$$

Fig. 13: The protocol to implement the Offline Phase: Part I 


\section{Protocol $\mathcal{P}_{\text {OffLine }}$ Part II}

$P_{1}$ Maps the $\ell$ and $t$ Values Using the Above Randomness.

- For $i \in\{1, \ldots, \operatorname{iw}(f)\}, P_{1}$ determines the sequence of switches involved in mapping the input label $\pi(i) \in \operatorname{ow}(f)$ to the output label $i \in \operatorname{iw}(f)$. Denote the sequence of switches by $\left(i_{1}, \ldots, i_{k}\right)$, and the index of the input wire the values goes through by $j_{1}, \ldots, j_{k}$. Note that $k=O(\log N), i_{k}=\lceil i / 2\rceil$, and $j_{k}=i \bmod 2$.

- $P_{1}$ then computes the following $d, m$ values and calls $($ input,$\cdot)$ of the $\mathcal{F}_{\mathrm{MPC}}$ on each to store the value in the functionality (i.e. share among the parties)

$$
\begin{aligned}
& d^{\ell, i}=h_{d}^{\ell, \pi(i)}+\sum_{j=1}^{k} u_{j_{k}}^{\ell, i_{j}} \doteq \ell_{\pi(i)}+\text { out }_{d, j_{k}}^{\ell, i_{k}}, \\
& m^{\ell, i}=h_{m}^{\ell, \pi(i)}+\sum_{j=1}^{k} v_{j_{k}}^{\ell, i_{j}} \doteq \mathrm{out}_{m, j_{k}}^{\ell, i_{k}}+d^{\ell, i} \cdot K, \\
& d^{t, i}=h_{d}^{t, \pi(i)}+\sum_{j=1}^{k} u_{j_{k}}^{t, i_{j}} \doteq t_{\pi(i)}+\text { out }_{d, j_{k}}^{t, i_{k}}, \\
& m^{t, i}=h_{m}^{t, \pi(i)}+\sum_{j=1}^{k} v_{j_{k}}^{t, i_{j}} \doteq \mathrm{out}_{m, j_{k}}^{t, i_{k}}+d^{t, i} \cdot K,
\end{aligned}
$$

Players Check $P_{1}$ 's Work and Compute $p_{i}, q_{i}$.

- For $i \in\{1, \ldots, \operatorname{iw}(f)\}$ players call $\mathcal{F}_{\text {MPC }}$ to compute (let $\left.j=i \bmod 2\right)$

$$
\left[n^{\ell, i}\right]=\left[\mathrm{out}_{m, j}^{\ell,[i / 2\rceil}\right]+\left[d^{\ell, i}\right] \cdot[K], \quad\left[n^{t, i}\right]=\left[\mathrm{out}_{m, j}^{t,\lceil i / 2\rceil}\right]+\left[d^{t, i}\right] \cdot[K] .
$$

- Parties then compute and open $\left[n^{\ell, i}-m^{\ell, i}\right]$ and $\left[n^{t, i}-m^{t, i}\right]$. If either is not 0 , players call Cheat(1) on the $\mathcal{F}_{\mathrm{MPC}}$ functionality. This will either abort, or return the input of $P_{1}$ (and hence the function), in the latter case the players can now proceed with evaluating the function using standard MPC and without the need for $P_{1}$ to be involved. If the opened value is zero the players compute and open

$$
\begin{aligned}
& {\left[p_{i}\right]=\left[r_{i}\right]-\left[d^{\ell, i}\right]+\left[\text { out }_{d, j}^{\ell,\lceil i / 2\rceil}\right] \doteq\left[r_{i}-\ell_{\pi(i)}\right],} \\
& {\left[q_{i}\right]=\left[s_{i}\right]-\left[d^{t, i}\right]+\left[\text { out }_{d, j}^{t,\lceil i / 2\rceil}\right]+p_{i} \cdot[K] \doteq\left[s_{i}-t_{\pi(i)}+p_{i} \cdot K\right],}
\end{aligned}
$$

Fig. 14: The protocol to implement the Offline Phase: Part II 


\section{Simulator $\mathcal{S}_{\text {OFFLINE }}$}

The protocol is described in the $\mathcal{F}_{\mathrm{MPC}}$-hybrid model, thus we only need to specify the simulator for the Input Function one. Let's denote the set of corrupted parties by $C \subset\left\{P_{1}, \ldots, P_{N}\right\}$.

\section{Input Function:}

$P_{1}$ Shares his Circuit/Function.

$-P_{1} \in C$ :

- Simulator $\mathcal{S}_{\text {Offune }}$ runs the protocol honestly and then waits for $P_{1}$ to broadcast $b_{i}$ for all $i \in$ $\{1, \ldots, N\}$ and $G_{j}$ for all $j \in\{1, \ldots, g\}$, he then sends them to ideal functionality $\mathcal{F}_{\text {Offline }}$.

- Simulator $\mathcal{S}_{\text {OffLINe }}$ evaluates $b_{i} \cdot\left(1-b_{i}\right)$ for all $i \in\{1, \ldots, N\}$ and similarly for $\left[G_{j}\right] \cdot\left(1-\left[G_{j}\right]\right)$ for $j \in\{1, \ldots, g\}$. If any of them is not 0 , simulator abort (since in this case $P_{1}$ has not entered a valid function).

$-P_{1} \notin C$ :

- Simulator $\mathcal{S}_{\text {OfFLINE }}$ generates a random circuit with $g$ gates $G_{j}^{\prime}$ for all $j \in\{1, \ldots, g\}$ and finds its corresponding mapping $\pi^{\prime}$. Then it determines a vector of selection bits $\left(b_{1}^{\prime}, \ldots, b_{N}^{\prime}\right)$ corresponding to the switching network representing the mapping $\pi^{\prime}$.

- $\mathcal{S}_{\text {Offline }}$ calls (input, $b_{i}^{\prime}$ ) for all $i \in\{1, \ldots, N\}$.

- $\mathcal{S}_{\text {Offline }}$ calls $\left(\right.$ input,$G_{j}^{\prime}$ ) for all $j \in\{1, \ldots, g\}$.

- Simulator $\mathcal{S}_{\text {OfFLINE }}$ evaluates $\left[b_{i}^{\prime}\right] \cdot\left(1-\left[b_{i}^{\prime}\right]\right)$ for all $i \in\{1, \ldots, N\}$ and similarly for $\left[G_{j}^{\prime}\right] \cdot\left(1-\left[G_{j}^{\prime}\right]\right)$ for $j \in\{1, \ldots, g\}$.

Players Generate Randomness for the Switching Network.

- Simulator $\mathcal{S}_{\text {Offune }}$ follows the protocol honestly.

$P_{1}$ Maps the $\ell$ and $t$ Values Using the Randomness.

- Simulator $\mathcal{S}_{\text {OFfLINE }}$ follows the protocol honestly.

Players Check $P_{1}$ 's Work and Compute $p_{i}, q_{i}$.

- Players follow the steps of protocol and simulator aborts if the checks were failed by any of players.

Fig. 15: Simulator $\mathcal{S}_{\text {OfFLINE }}$ 
The protocol is described in the $\mathcal{F}_{\text {OfFLINE }}$-hybrid model.

Input Function: If $P_{1} \notin C$, simulator generates a random circuit with $g$ gates and corresponding mapping $\pi^{\prime}$, and follows the protocol honestly. If $P_{1} \in C$, simulator $\mathcal{S}_{\text {ONLINE }}$ runs the protocol honestly and then waits for $P_{1}$ to broadcast $\pi$ and $f$, he then sends them to ideal functionality $\mathcal{F}_{\text {ONLINE }}$.

Input Data: If $P_{i} \notin C$, simulator generates a dummy input $x_{i}^{\prime}$ and follows the steps of protocol honestly. If $P_{i} \in C$, simulator runs the protocol honestly and waits for them to send their input to $\mathcal{F}_{\text {Offuine }}$, he then sends them to $\mathcal{F}_{\text {ONLINE }}$ ideal functionality.

Output: Simulator follows the protocol steps honestly. For $P_{i} \notin C$

\section{- Preparing Inputs to the Circuit:}

- Simulator follows the steps of protocol honestly.

- Evaluating the Circuit: For every gate $1 \leq i \leq g$ in the circuit players execute the following (here we assume that the gates are indexed in the same topological order $P_{1}$ chose to determine $\pi$ ):

- $P_{1}$ Prepares the Two Inputs for Gate $i$.

* Simulator follows the steps of protocol honestly.

- Players Check $P_{1}$ 's Input Preparation.

* Simulator follows the steps of protocol honestly and aborts if the checks are failed.

- Players Jointly Evaluate Gate $i$.

* The players store the value $\left[y_{i_{j}}\right]=d_{i_{j}}-\left[r_{i_{j}}\right]$ in the $\mathcal{F}_{\mathrm{MPC}}$ functionality.

* The $\mathcal{F}_{\text {MPC }}$ functionality is then executed so as to compute the output of the gate as

$$
\left[z_{i}\right]=\left(1-\left[G_{i}\right]\right) \cdot\left(\left[y_{i_{0}}\right]+\left[y_{i_{1}}\right]\right)+\left[G_{i}\right] \cdot\left[y_{i_{0}}\right] \cdot\left[y_{i_{1}}\right] .
$$

* Note that the outgoing wire label corresponding to the output wire of the $i$ th gate is $j=n+i$ (the first $n$ outgoing wires are input wires, hence output wire of the $i$ th gate is indexed $n+i$ ) so we just relabel $\left[z_{i}\right]$ to $\left[z_{j}\right]$.

* The players compute via the MPC functionality $\left[u_{j}\right]=\left[z_{j}\right]+\left[\ell_{j}\right]$.

* The players call (Output, $u_{j}$ ) so as to obtain $u_{j}$.

* The players then compute via the MPC functionality

$$
\left[v_{j}\right]=\left[t_{j}\right]+u_{j} \cdot[K]=\left[t_{j}+\left(z_{j}+\ell_{j}\right) \cdot K\right] .
$$

* The players call (Output, $v_{j}$ ) so as to obtain $v_{j}$. If $j$ is the output wire, simulator adjusts his share of output in the ideal execution to make the output consistent with the shares of honest parties as follows: suppose the output of that wire using the dummy values is $z_{i}$ and the output returned by the $\mathcal{F}_{\text {ONLINE }}$ ideal functionality is $z_{i}^{\prime}$, he then adds $z_{i}-z_{i}^{\prime}$ to the share of adversary $\left[z_{i}^{\prime}\right]$ in the ideal execution.

Fig. 16: The Protocol for implementing PFE 
To see that the simulated and real processes cannot be distinguished, we will show that the view of the environment in the ideal process is statistically indistinguishable from the view in the real process. This view consists of the corrupt players' view of the protocol execution as well as the inputs and outputs of honest players. The view of adversary includes $\left[u_{i}\right],\left[v_{i}\right], d_{i_{j}}, m_{i_{j}}, n_{i_{j}},\left[z_{i}\right]$ and if $i$ is the index of output wire, $z_{i}$. The shared values all look random and therefore are indistinguishable between ideal and real execution.

We next show that $d_{i_{j}}, m_{i_{j}}$ have uniform distribution. Observe that $u_{i}$ is blinded using the random value of input wires which is shared and therefore acts as a one-time pad, and as $P_{1}$ prepares the two inputs, it maintains the uniform distribution. Furthermore, $p_{i_{j}}$ also has uniform distribution from the security of offline protocol. The value $s_{i_{j}}$ acts as a one-time pad which is shared between the players and therefore, $m_{i_{j}}$ has a uniform distribution. In the ideal functionality we also have a uniform distribution, and as a result ideal and real are indistinguishable to the environment $\mathcal{Z}$.

For a malicious $P_{1}$, the distributions are the same, but we have to make sure that he has performed the input preparation correctly. In the next phase players check the $P_{1}$ 's computation. Player $P_{1}$ cheating means he has not calculated $d_{i_{j}}, m_{i_{j}}$ correctly. For him to be successful, he has to somehow adjust $n_{i_{j}}$ and $m_{i_{j}}$ to be equal. He only has a option to adjust $d_{i_{j}}$ and his share of $\left[S_{i_{j}}\right]$ to make the equality hold. Since he does not know $K$, the value $d_{i_{j}} \cdot K$ has a uniform distribution, and therefore the probability of him modifying $\left[S_{i_{j}}\right]$ to make the equality hold is equivalent to guessing $K$ and hence exponentially small in length of $K$. It follows that with overwhelming probability after the check the $P_{1}$ 's computation has been done correctly. If any check fails the simulator aborts and stop.

The final result $z_{i}$ is a secret shared value and as result has a uniform distribution. For the output wires, players open their share, and $z_{i}$ is learnt by all parties. In order to make the distribution of outputs indistinguishable, the simulator has to modify his share of $z_{i}$ in the ideal execution. He is able to do so and produce the exact same output for the ideal execution as described in Figure 16 . This completes the proof. 